\title{
Possible existence of a band of extended states induced by inter-Landau-band mixing in a quantum Hall system
}

\author{
Gang Xiong ${ }^{1}$, Shi-Dong Wang ${ }^{2}$, Qian Niu ${ }^{3,4}$, Yupeng Wang ${ }^{4}$ X C Xie ${ }^{4,5}$, \\ De-Cheng $\operatorname{Tian}^{6}$ and X R Wang ${ }^{2}$ \\ ${ }^{1}$ Physics Department, Beijing Normal University, Beijing 100875, People's Republic of China \\ ${ }^{2}$ Physics Department, Hong Kong University of Science and Technology, Clear Water Bay, \\ Hong Kong SAR, People's Republic of China \\ ${ }^{3}$ Physics Department, The University of Texas at Austin, Austin, TX 78712-1081, USA \\ ${ }^{4}$ International Centre for Quantum Structures, Institute of Physics, Chinese Academy of Sciences, \\ Beijing 100080, People's Republic of China \\ ${ }^{5}$ Physics Department, Oklahoma State University, Stillwater, OK 74078, USA \\ ${ }^{6}$ Physics Department, Wuhan University, Wuhan 430072, People's Republic of China
}

Received 29 August 2005

Published 27 January 2006

Online at stacks.iop.org/JPhysCM/18/2029

\begin{abstract}
The mixing of states with opposite chiralities in a quantum Hall system is shown to have a delocalization effect. It is possible that extended states may form bands because of this mixing, as is shown through a numerical calculation on a two-channel network model. Based on this result, a new phase diagram with a narrow metallic phase separating two adjacent $\mathrm{QH}$ phases and/or separating a QH phase from the insulating phase is proposed. The data from recent non-scaling experiments are reanalysed and it is shown that they seem to be consistent with the new phase diagram. However, due to finite-size effects, further study on large system size is still needed to conclude whether there are extended state bands in the thermodynamic limit.
\end{abstract}

\section{Introduction}

According to the scaling theory of localization [1], all electrons in a disordered twodimensional system are localized in the absence of a magnetic field. In the presence of a strong magnetic field $B$, a series of disorder-broadened Landau bands (LBs) will appear, and an extended state resides at the centre of each band while states at other energies are localized [2]. The integrally quantized Hall plateaus (IQHPs) are observed when the Fermi level lies in localized states, with the value of the Hall conductance, $\sigma_{x y}=n e^{2} / h$, related to the number of occupied extended states $(n)$. Many previous studies [3-23] have been focused on so-called plateau transitions. The issue there is how the Hall conductance jumps from one quantized value to another when the Fermi level crosses an extended state. There are two 
competing proposals. One is the global phase diagram [5] based on the levitation of extended states conjectured by Khmelnitskii [24] and Laughlin [25]. A crucial prediction of this phase diagram is that an integer quantum Hall effect (IQHE) state $n$ in general can only go into another IQHE state $n \pm 1$, and that a transition into an insulating state is allowed only from the $n=1$ state. The other is the so-called direct transition phase diagram [6] in which transitions from any IQHE state to the insulating phase are allowed when the disorder is increased at fixed $B$. So far, most experiments $[17,18]$ are consistent with the direct transition phase diagram although the early experiments were interpreted in terms of the global phase diagram.

One important yet overlooked issue regarding IQHE is the nature of both plateauplateau and plateau-insulator transitions. In all existing theoretical studies, these transitions are assumed to be continuous quantum phase transitions. This assumption is mainly due to the early scaling experiments $[3,4]$. The fingerprint of a continuous phase transition is scaling laws around the transition point. In the case of the IQHE, it means algebraic divergence of longitudinal resistance slope and algebraic shrinkage of longitudinal resistance peak width in temperature $T$ at the transition point. However, some experiments show that the longitudinal resistance slope remains finite [21] and the resistance peak width remains nonzero $[22,23]$ when extrapolated to zero temperature. This implies a non-scaling behaviour around a transition point, contradicting the expectation of continuous quantum phase transitions suggested by the theories. Thus the nature of these transitions should be re-examined.

The samples used in the non-scaling experiments [21] are relatively dirty, and strong disorders should lead to a strong inter-Landau-band mixing. In a recent letter [26], we showed that the single extended state at each LB centre broadens into a narrow band of extended states when the interband mixing of opposite chirality is taken into account. A narrow metallic phase exists between two adjacent IQHE phases and between an IQHE phase and the insulating phase. A plateau-plateau or plateau-insulator transition corresponds to two consecutive quantum phase transitions instead of one as suggested by existing theories. This possibility has usually been overlooked in previous studies where each longitudinal conductance peak is related to a single extended state [16]. In this paper we shall present the detailed description of this study.

The paper is organized as follows. The semiclassical network model for two coupled LBs is illustrated in section 2. It is shown that mixing of localized states of opposite chirality tends to delocalize a state while mixing of states of the same chirality does not. The similarities and differences between the case we consider and models for double-layer systems and spindegenerate systems are also discussed. Our approach, the level-statistics technique, is described in section 3. Section 4 has four subsections containing our numerical results, conclusions and discussions. Section 4.1 provides our main numerical results and conclusions. In section 4.2, possible finite size effects and a theoretical understanding for non-scaling behaviours based on standard scaling theory $[27,28]$ are discussed. Section 4.3 gives further numerical results which directly show a narrow band of extended states in several cases, and a new phase diagram of quantum Hall systems is proposed. To further support our results, in section 4.4 we reanalyse the original data from the non-scaling experiments [21] and show that the nonscaling behaviours in each IQHP-insulator transition can be attributed to two quantum phase transition points. Recent experiments [29-33], in which scaling behaviours are observed, are also discussed and shown to be not inconsistent with early non-scaling experiments and our numerical results. The conclusions of this paper are summarized in section 5 .

\section{The semiclassical model including inter-Landau-band mixing}

According to the semiclassical theory [34], the motion of an electron in a strong magnetic field and in a smooth random potential can be decomposed into a rapid cyclotron motion and a slow 
drifting motion of its guiding centre. The kinetic energy of the cyclotron motion is quantized by $E_{n}=(n+1 / 2) \hbar \omega_{\mathrm{c}}$, where $\omega_{\mathrm{c}}$ is the cyclotron frequency and $n$ the LB index. The trajectory of the drifting motion of the guiding centre is thus along an equipotential contour of value $V_{0}=E-E_{n}$, where $E$ is the total energy of the electron. The local drifting velocity $\vec{v}(\vec{r})$ is determined by (in SI units)

$$
\vec{v}(\vec{r})=\nabla V(\vec{r}) \times \vec{B} /\left(e B^{2}\right)
$$

where $\nabla V(\vec{r})$ is the local potential gradient. The equipotential contour consists of many closed loops. Neglecting quantum tunnelling effects, each loop corresponds to trajectory of one eigenstate. The motion of electrons is thus confined around these loops with deviations typically of the order of the cyclotron radius $l_{\mathrm{c}}=\sqrt{\hbar /(e B)}$.

To illustrate this semiclassical picture, let us think of the smooth random potential as a landscape of many peaks and valleys distributed randomly in the plane. Imagine that the landscape is filled with water up to a height of value $V_{0}$. The equipotential contour of value $V_{0}$ is the boundary of land and water. According to the percolation theory [35], the percolation threshold of a two-dimensional (2D) continuum model is $p_{\mathrm{c}}=1 / 2$, where $p_{\mathrm{c}}$ is the occupation probability of the medium (the land or the water). For simplicity, we suppose that the distribution of the random potential is symmetric around zero. By symmetry the percolation point of both the land and the water is at $V_{0}=0$ in this case. When $V_{0}<0$, the occupation probability of land is above $1 / 2$. Thus the land percolates and the water forms isolated lakes. These lakes are around valleys and their boundaries correspond to trajectories of localized states. In the case of $V_{0}>0$, the water forms a percolating sea and the land becomes isolated islands around potential peaks. The boundary of each island is an electronic state. In short, semiclassical electronic states in a $\mathrm{QH}$ system are equipotential loops. These loops are localized around potential peaks for $V_{0}>0$ and around potential valleys for $V_{0}<0$. The drifting direction of each loop is unidirectional. This means that they are chiral states. From equation (1) one can see that states around peaks have opposite chirality from states around valleys because the directions of the local potential gradient around a peak are opposite to that around a valley. If one views the plane from the direction opposite to the magnetic field, the drifting is clockwise around valleys and anticlockwise around peaks, as shown in figure 1 . Right at $V_{0}=0$ both the land and the water percolate, and the intersection between them is the trajectory of an extended state. This means that there is only one extended state at $V_{0}=0$ for each LB. As $V_{0}$ approaches zero from both sides, the localization length $\xi$ of the system tends to diverge as

$$
\xi \propto\left|V_{0}\right|^{-v}
$$

where the critical exponent $v=4 / 3$ according to the classical percolation theory. Quantum effects are ignored in the above semiclassical argument. When two spatially separated loops on the same equipotential contour come close at saddle points of the random potential, quantum tunnellings should be considered. An example in the case of $V_{0}<0$ is shown in figure 1(a). In the absence of interband mixing, numerical calculations have suggested that there is still only one extended state in each LB while the value of the critical exponent $v$ is modified to be around $7 / 3$ [10].

In the case when the width of the LBs is comparable with the spacing between adjacent LBs (the Landau gap), inter-Landau-band mixing should no longer be ignored. In order to investigate the consequences of inter-Landau-band mixing, we shall consider a simple system of two adjacent LBs. Since we are interested in interband mixing of opposite chirality, we consider those states with energy between the lower and the upper bands whose centres are at $E_{1}$ and $E_{\mathrm{u}}$, respectively, as shown in figure 2(a). Thus, equipotential loops are $V_{1}=E-E_{1}>0$ and $V_{\mathrm{u}}=E-E_{\mathrm{u}}<0$ for the lower and the upper LBs, respectively. Using the semiclassical 


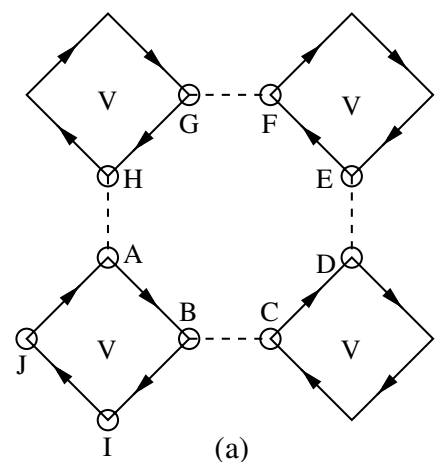

(a)

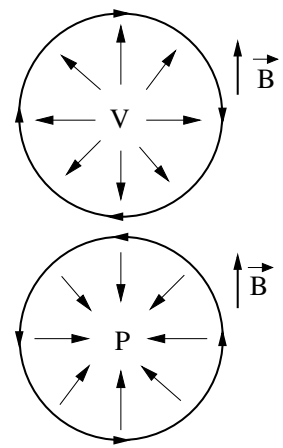

(b)

Figure 1. (a) Four neighbouring loops in a one-band model for the case of $V_{0}<0$. Dashed lines denote quantum tunnellings. The arrows indicate the drifting direction. (b) Loops localized around a valley and a peak, respectively. The arrows inside a loop show the directions of local potential gradient around the peak or valley. The arrows on a loop indicate the drifting direction.

theory described in the previous paragraphs, states from the upper band should move along equipotential loops around potential valleys while those from the lower band around potential peaks as shown in figure 2(b). The loops for the upper band drift in the clockwise direction, and those for the lower band in the anticlockwise direction. These two sets of loops are thus spatially separated and have opposite chirality. If we assume that peaks and valleys of random potential form two coupled square lattices, the loops can be arranged as shown in figure 3(a), where $\mathrm{P}$ and $\mathrm{V}$ denote peaks and valleys, respectively. In the absence of interband mixing, the model is reduced to two decoupled single-band models and all electronic states between the two LBs are localized. If we introduce interband mixing, the localized loops may become less localized. To see this, let us consider an extreme case with no tunnellings at saddle points, but with such a strong interband mixing that an electron will move from a loop around a valley to its neighbouring loop around a peak and vice versa, as shown by $\mathrm{B} \rightarrow \mathrm{C}$ in figure 3(a). Follow the trajectory of an electron starting at $\mathrm{A}$; it will be $\mathrm{A} \rightarrow \mathrm{B} \rightarrow \mathrm{C} \rightarrow \mathrm{D} \rightarrow \mathrm{E} \cdots$. The electron is no longer confined on a closed loop, but delocalized!

Before going on, we would like to give a short discussion on the relation between large disorder magnitude and weak magnetic field. The above semiclassical picture for the network model is valid only when the typical fluctuation length of random potential, denoted by $L_{\mathrm{F}}$, is much larger than the magnetic length $l_{\mathrm{c}}=\sqrt{\hbar /(e B)}$. Let us take $L_{\mathrm{F}}$ as a large fixed value. Then, the effect of inter-band mixing is controlled mainly by two parameters. One 

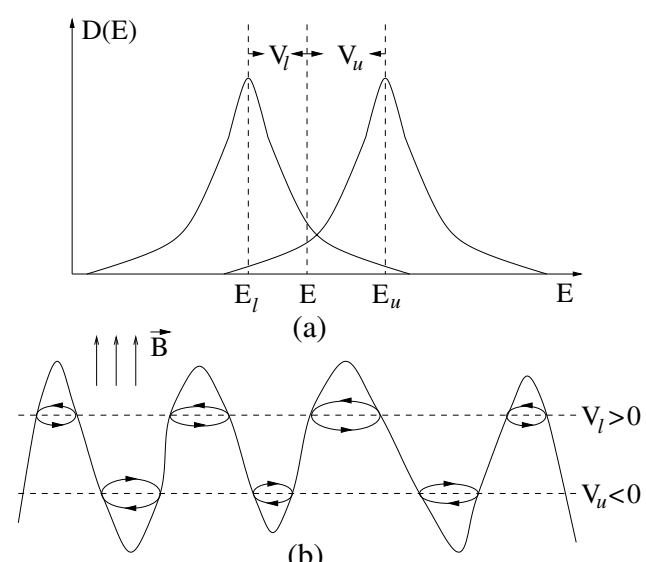

(b)

Figure 2. (a) Two adjacent Landau bands in the case when the disorder broadened band width is comparable with the Landau gap. $D(E)$ is the density of states. $E_{\mathrm{u}}$ and $E_{1}$ denote the centres of the two bands. (b) Schematic plot of the two sets of equipotential loops on the two-dimensional random potential for electronic states of energy $E$ shown in (a). It is the projection of a 3D landscape on a $2 \mathrm{D}$ vertical plane. The solid curve represents the schematic plot of the $2 \mathrm{D}$ random potential. Two dashed horizontal lines indicate two constant potential planes $V(\vec{r}) \equiv V_{0}$, one for the lower band with $V_{0}=V_{1}=E-E_{\mathrm{u}}>0$ and the other for the upper band with $V_{0}=V_{\mathrm{u}}=E-E_{1}<0$. The ellipses denote the loops where the two constant potential planes intersect with the $2 \mathrm{D}$ random potential. Arrows on the loops show drifting directions. $\vec{B}$ is the magnetic field.

is the ratio of disorder magnitude $W$ and Landau gap $\hbar \omega_{\mathrm{c}}$, which is proportional to $W / B$ and determines whether inter-band mixing needs to be considered. The other is the ratio of the magnetic length $l_{\mathrm{c}}$ and the typical distance between an upper-band loop and its neighbouring lower-band loop $L_{\mathrm{F}} \frac{\hbar \omega_{\mathrm{c}}}{W}$, which is proportional to $W / B^{3 / 2}$ and determines the typical strength of inter-band mixing in the network model. Therefore, the cases of strong disorder $W$ and weak magnetic field $B$ are, to some extent, equivalent in the network model since both of them lead to large inter-band mixing. Of cause, values of local potential gradients are also important, since they determine the detailed distribution of inter-band mixing in the model.

In the one-band model, an electron can also hop from one loop to its neighbouring loops by quantum tunnellings. At a first glance, this effect seems similar to that of interband mixing. However, they are fundamentally different. In the one-band model, electronic states for a given $V_{0}$ have the same chirality. Thus the drifting direction of an electron will be inverted when it tunnels into neighbouring loops. This means that a strong tunnelling in a one-band model will induce an effective backward-scattering that tends to also localize electrons. We can understand this by considering a small part of the one-band model as shown in figure 1(a) where all loops are moving in a clockwise direction. Without tunnelling, the trajectory of an electron starting from point $\mathrm{A}$ is $\mathrm{A} \rightarrow \mathrm{B} \rightarrow \mathrm{I} \rightarrow \mathrm{J} \rightarrow \mathrm{A}$, a clockwise closed loop. With strong tunnellings, the trajectory will tend to be $\mathrm{A} \rightarrow \mathrm{B} \rightarrow \mathrm{C} \rightarrow \mathrm{D} \cdots \rightarrow \mathrm{H} \rightarrow \mathrm{A}$, an anticlockwise closed loop. Thus, the tunnellings between loops of the same chirality cannot delocalize states.

The two-channel CC model can also be used to simulate both spin-degenerate and bilayer systems. The model for the spin-degenerate system is the same as we use. The only difference is that loops for spin-up and spin-down states are present at the same positions in real space, and the chiralities of loops of spin-up and spin-down states are the same, while the main difference between the model for the bilayer system and ours is that the real space distributions of random potentials in the two layers are different. 

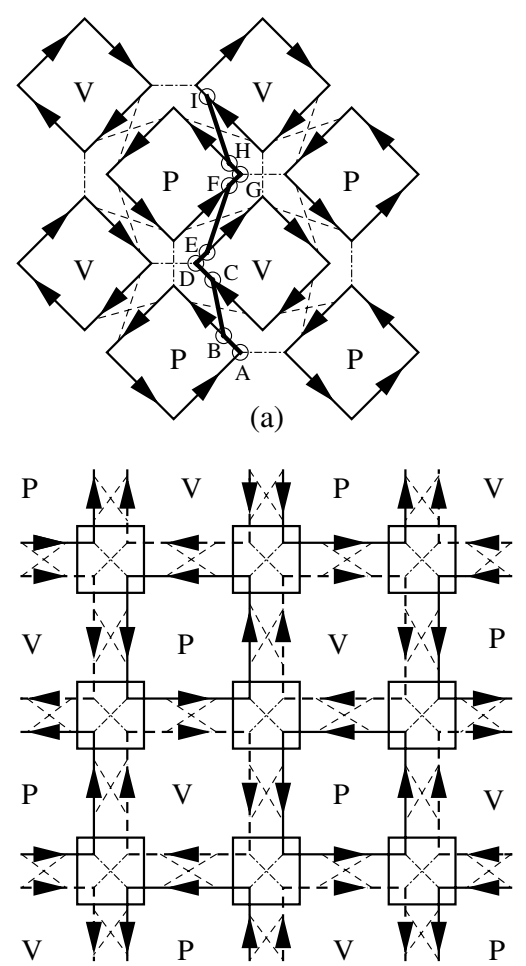

(b)

Figure 3. (a) Topological plot of the trajectories of the drifting motion of guiding centres (rhombus). The drifting motion around a potential peak (valley) is denoted by $\mathrm{P}(\mathrm{V})$, and their directions are indicated by the arrows. Dashed lines stand for interband mixing, and dash-dotted lines for tunnelling at saddle points. The thick line (A-I) describes the trajectory of an electron due to a strong interband mixing. (b) The equivalent two-channel network model of (a). Solid and dashed lines on each link denote two channels from two LBs. Squares stand for saddle points. P, V and arrows have the same meaning as those in (a).

It is worthwhile to explain why we consider only those states between two LB centres. For states outside this range, both sets of loops are localized around either valleys or peaks. This means that interband mixing mainly occurs between two loops localized around the same position, and this mixing will not delocalize a state. In fact, as explained in the previous paragraph, the mixing of the same chirality does not help delocalizing an electron. This is why we shall consider mixing between spatially separated states with opposite chirality. Of course, it does not mean that the mixing of the same chirality has no effect at all. As it was found in some previous works [7], this kind of mixing may shift an extended state from its LB centre. Level shifting due to mixing between states of the same chirality may distort the shape of the phase diagram, but should not alter its topology. The emergence of the bands of extended states is exclusively due to the mixing between states of opposite chirality.

Now, we describe our two-channel network model in detail. Assume that tunnellings of two neighbouring localized states (loops) of the same band occur around saddle points, and interband mixing takes place only on the links; figure 3(a) is topologically equivalent to the model shown in figure 3(b). Figure 3(b) is the schematic illustration of our twochannel Chalker-Coddington network model. It is similar to the model studied in previous publications [36, 37]. There are two channels on each link. One, denoted by a solid line, is 


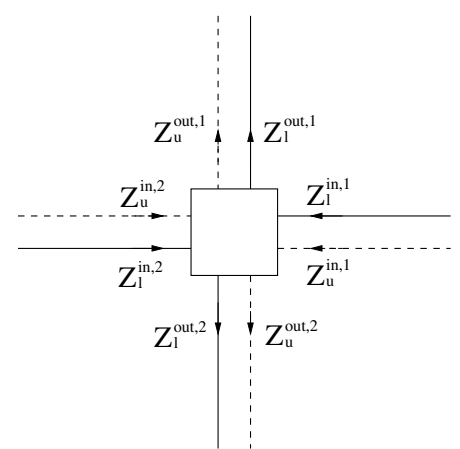

(a)

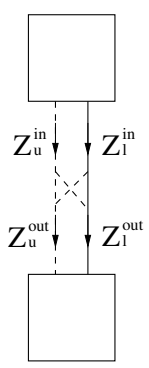

(b)

Figure 4. (a) A node with four incoming and four outgoing channels. $Z_{\mathrm{u}(1)}^{\mathrm{in}, i}$ is the wavefunction amplitude of the $i$ th incoming wave from the upper (lower) LB. $Z_{\mathrm{u}(\mathrm{l})}^{\mathrm{out} i}$ is that of outgoing wavefunction amplitude. (b) A link with two channels. $Z_{\mathrm{u}(\mathrm{l})}^{\text {in (out) }}$ is the incoming (outgoing) wavefunction amplitude of the upper (lower) LB.

from the lower LB around a potential peak. The other (dashed line) is from the upper LB moving around a potential valley. The arrows indicate the drifting direction of the two sets of states. At each node, the tunnelling between two neighbouring states of the same LB occurs. As shown in figure $4(\mathrm{a})$, let $Z_{\mathrm{u}(\mathrm{l})}^{\mathrm{in}, 1}$ and $Z_{\mathrm{u}(\mathrm{l})}^{\mathrm{in}, 2}$ be the incoming wave amplitudes of states 1 and 2 from upper (lower) LB, respectively, and $Z_{\mathrm{u}(\mathrm{l})}^{\mathrm{out}, 1}$ and $Z_{\mathrm{u}(\mathrm{l})}^{\mathrm{out}, 2}$ be the outgoing wave amplitudes of the two states. The tunnelling is described by an $S O(4)$ matrix

$$
\left(\begin{array}{c}
Z_{\mathrm{u}}^{\text {out }, 1} \\
Z_{\mathrm{u}}^{\text {out }, 2} \\
Z_{1}^{\text {out }, 1} \\
Z_{1}^{\text {out }, 2}
\end{array}\right)=\left(\begin{array}{cccc}
s_{\mathrm{u}}^{\mathrm{R}} & s_{\mathrm{u}}^{\mathrm{L}} & 0 & 0 \\
-s_{\mathrm{u}}^{\mathrm{L}} & s_{\mathrm{u}}^{\mathrm{R}} & 0 & 0 \\
0 & 0 & s_{1}^{\mathrm{R}} & s_{1}^{\mathrm{L}} \\
0 & 0 & -s_{1}^{\mathrm{L}} & s_{1}^{\mathrm{R}}
\end{array}\right)\left(\begin{array}{c}
Z_{\mathrm{u}}^{\text {in, }} \\
Z_{\mathrm{u}}^{\text {in, } 2} \\
Z_{1}^{\text {in, }, 1} \\
Z_{1}^{\text {in, }, 2}
\end{array}\right),
$$

where the subscripts $u$ and 1 denote the upper and the lower bands, respectively. The elements $s_{\mathrm{u}(\mathrm{l})}^{\mathrm{L}}$ and $s_{\mathrm{u}(\mathrm{l})}^{\mathrm{R}}$ are tunnelling coefficients of an incoming wavefunction in the upper (lower) band being scattered into outgoing channels on its left-hand and right-hand sides, respectively. $s_{\mathrm{u}(1)}^{\mathrm{R}}$ and $s_{\mathrm{u}(\mathrm{l})}^{\mathrm{L}}$ are related to each other as $s_{\mathrm{u}(\mathrm{l})}^{\mathrm{R}}=\sqrt{1-\left(s_{\mathrm{u}(\mathrm{l})}^{\mathrm{L}}\right)^{2}}$ due to the orthogonality of the matrix. Under the quadratic potential barrier approximation-i.e., $V(x, y)=-U x^{2}+U y^{2}+V_{\mathrm{c}}$ around a saddle point, where $U$ is a constant describing the strength of potential fluctuation and $V_{\mathrm{c}}$ is the potential barrier at the point-one can show that the left-hand scattering amplitude is given by [38]

$$
s_{\mathrm{u}(\mathrm{l})}^{\mathrm{L}}=\left[1+\exp \left(-\pi \epsilon_{\mathrm{u}(\mathrm{l})}\right)\right]^{-1 / 2},
$$

where $\epsilon_{\mathrm{u}(\mathrm{l})}=\left[E+V_{\mathrm{c}}-\left(n_{\mathrm{u}(\mathrm{l})}+1 / 2\right) E_{2}\right] / E_{1}$ with $E$ being electron energy, $E_{1}=\frac{\hbar \omega_{\mathrm{c}}}{2 \sqrt{2}} \sqrt{K-1}$, $E_{2}=\frac{\hbar \omega_{\mathrm{c}}}{\sqrt{2}} \sqrt{K+1}$, and $K=\sqrt{\frac{64 U^{2}}{m^{2} \omega_{\mathrm{c}}^{4}}+1}$. The energies of the cyclotron motion in the two bands are $\left(n_{\mathrm{u}}+1 / 2\right) E_{2}$ and $\left(n_{1}+1 / 2\right) E_{2}$, respectively, where $n_{\mathrm{u}(\mathrm{l})}$ are the band indices and $\Delta n=n_{\mathrm{u}}-n_{\mathrm{l}}=1$. The dimensionless ratio $E_{r}=E_{2} / E_{1}=2 \sqrt{1+\frac{2}{K-1}}$ approaches two from above as $U$ or the inverse of $\omega_{\mathrm{c}}$ increases [38], i.e., the strong disorder regime or a weak magnetic field. In our calculations, we choose it to be 2.2 since this is the regime we are interested in. For convenience, we choose $E_{2}$ as the energy unit and the cyclotron energy of 
the lower band as the reference point. The energy regime between the two band centres is thus $E \in[0,1]$.

Inter-band mixing between two channels on a link as shown in figure 4(b) is described by a $U(2)$ matrix

$$
\begin{aligned}
& \left(\begin{array}{c}
Z_{1}^{\text {out }} \\
Z_{\mathrm{u}}^{\text {out }}
\end{array}\right)=M\left(\begin{array}{c}
Z_{1}^{\text {in }} \\
Z_{\mathrm{u}}^{\text {in }}
\end{array}\right), \\
& M=\left(\begin{array}{cc}
\mathrm{e}^{\mathrm{i} \phi_{1}} & 0 \\
0 & \mathrm{e}^{\mathrm{i} \phi_{2}}
\end{array}\right)\left(\begin{array}{cc}
\cos \theta & \sin \theta \\
-\sin \theta & \cos \theta
\end{array}\right)\left(\begin{array}{cc}
\mathrm{e}^{\mathrm{i} \phi_{3}} & 0 \\
0 & \mathrm{e}^{\mathrm{i} \phi_{4}}
\end{array}\right),
\end{aligned}
$$

where $\sin \theta$ describes the interband mixing. $\phi_{i}(i=1-4)$ are random Aharonov-Bohm phases accumulated along propagation paths. In our calculations, we shall assume that they are uniformly distributed in $[0,2 \pi]$ [34]. In the following discussion, a parameter $J$, defined as $\sqrt{J /(1+J)}=\sin \theta$, is used to characterize the mixing strength. $J$ will take the same value for all links in our calculations. We hope that this simplification will not affect the physics.

\section{The application of the level-statistics technique on the network model}

Electron localization length is often obtained from the transfer matrix method. For a twodimensional system, however, it is well known that this quantity alone does not provide conclusive answers to questions related to the metal-insulator transition (MIT) [39]. On the other hand, level-statistics analysis has been used in studying MIT [40, 41]. Level-statistics analysis is based on random matrix theory (RMT) [42]. The basic idea is that the localization property of an electronic state can be determined by the statistical distribution function $P(s)$ of the spacing $s$ of two neighbouring levels. For localized states, the distribution is Poissonian $P_{\mathrm{PE}}(s)=\exp (-s)$, called a 'Poissonian ensemble (PE)'. In the case of extended states, the nearest neighbour level spacing distribution has the following form [42]:

$$
P(s)=C_{1}(\beta) s^{\beta} \exp \left[-C_{2}(\beta) s^{2}\right]
$$

where $C_{1}(\beta)$ and $C_{2}(\beta)$ are normalization factors determined by $\int P(s) \mathrm{d} s=1$ and $\int s P(s) \mathrm{d} s=1$. The parameter $\beta$ is determined by the dynamical symmetry of the system. The case of $\beta=1$ is for systems with time-reversal symmetry and an integer total angular momentum and is referred to as a 'Gaussian orthogonal ensemble'. Systems with time-reversal symmetry and a half-integer total angular momentum belong to the case of $\beta=4$, called a 'Gaussian symplectic ensemble'. For systems without time-reversal symmetry $\beta=2$, and it is called the 'Gaussian unitary ensemble (GUE)'. A fundamental difference between the level statistics property of extended states and localized states is that $P(s)$ at $s=0$ is zero for extended states and one for localized states. The physical reason for this difference is the socalled 'level repulsion' of extended states. Two extended states with the same 'bare energy' will overlap in real space (since they are extended in real space) and form two new extended states with different energies, while localized states can have the same energy staying in different regions of real space. This approach is appropriate for the network model of quantum Hall systems because localized states in the model are loops in different regions of real space while extended states in the model are formed by quantum percolation of such localized loops.

We shall follow the approach proposed by Klesse and Metzler [43]. A quantum state of a network model can be expressed by a vector whose components are electronic wavefunction amplitudes on the links. In our case, the vector can be written as $\Phi=\left(\left\{Z_{\mathrm{u}}^{i}, Z_{1}^{i}\right\}\right)$, where $Z_{\mathrm{u}}^{i}$ and $Z_{1}^{i}$ are the electron wavefunction amplitudes of the upper band $(u)$ and the lower band $(l)$ on the $i$ th link, respectively. As shown by Fertig [44], the network model can be described by an evolution operator $\hat{U}(E)$, an $E$-dependent matrix determined by the scattering properties 
of nodes and links in the model. (As an example, the evolution operator of a two-channel network of size $L=2$ with periodic boundaries on both directions is constructed explicitly in the appendix.) In general, the eigenvalue equation of the evolution operator is

$$
\hat{U}(E) \Phi_{\alpha}(E)=\mathrm{e}^{\mathrm{i} \omega_{\alpha}(E)} \Phi_{\alpha}(E),
$$

where $\alpha$ is the eigenstate index of $\hat{U}$. The true eigenenergies $\left\{E_{n}\right\}$ of the system are those energies at which $\omega_{\alpha}(E)$ is an integer multiple of $2 \pi$. It has been shown by Klesse and Metzler [43] that the set of quasi-energies $\left\{\omega_{\alpha}(E)\right\}$ corresponds to the excitation spectrum of the stationary state with energy $E$. Therefore, the statistics property of the set of quasienergies $\left\{\omega_{\alpha}(E)\right\}$ at $E$ is the same as that of true eigenenergies $\left\{E_{n}\right\}$ around $E$, and the localization property of an electronic state with an energy $E$ can be obtained by the quasienergies. The advantage of this approach is that all the quasi-energies can be used in the analysis so that better statistics can be obtained.

Chalker and Coddington [34] showed numerically that an open boundary condition along one direction creates extended edge states along the other direction. In order to get rid of the edge states, we employ a periodic boundary along both directions in our calculation. For a two-channel network model of $L \times L$ nodes with periodic boundaries along both directions, there are $4 L^{2}$ components in $\Phi . \hat{U}$ is thus a $\left(4 L^{2}\right) \times\left(4 L^{2}\right)$ matrix. However, there is a special property of the network model [45]: the nodes scatter electrons only from vertical channels into horizontal channels and vice versa. If one separates $\Phi$ into the set of wavefunction amplitudes on the horizontal links $\Phi_{\mathrm{H}}$ and the set of wavefunction amplitudes on the vertical links $\Phi_{\mathrm{V}}$, the evolution equation in one time step can be written in the following form:

$$
\left(\begin{array}{l}
\Phi_{\mathrm{H}}(t+1) \\
\Phi_{\mathrm{V}}(t+1)
\end{array}\right)=\left(\begin{array}{cc}
\hat{0} & \hat{U}_{\mathrm{V} \rightarrow \mathrm{H}} \\
\hat{U}_{\mathrm{H} \rightarrow \mathrm{V}} & \hat{0}
\end{array}\right)\left(\begin{array}{l}
\Phi_{\mathrm{H}}(t) \\
\Phi_{\mathrm{V}}(t)
\end{array}\right),
$$

where $\hat{0}$ is the $\left(2 L^{2}\right) \times\left(2 L^{2}\right)$ zero matrix. $\hat{U}_{\mathrm{V} \rightarrow \mathrm{H}}$ describes how the wavefunction on the vertical links evolves into that on the horizontal links. Similarly, $\hat{U}_{\mathrm{H} \rightarrow \mathrm{V}}$ describes that from horizontal to vertical links. For the detailed derivation, we refer readers to the example shown in the appendix. The evolution equation in two time steps is given as

$$
\begin{aligned}
& \Phi_{\mathrm{H}}(t+2)=\hat{U}_{\mathrm{V} \rightarrow \mathrm{H}} \hat{U}_{\mathrm{H} \rightarrow \mathrm{V}} \Phi_{\mathrm{H}}(t) \\
& \Phi_{\mathrm{V}}(t+2)=\hat{U}_{\mathrm{H} \rightarrow \mathrm{V}} \hat{U}_{\mathrm{V} \rightarrow \mathrm{H}} \Phi_{\mathrm{V}}(t) .
\end{aligned}
$$

Therefore, the evolution matrix in two time steps is block diagonal and the two blocks have essentially the same statistical property. We thus need only consider one of them.

We study the model for $L=8,12,16,20$ and 24. The calculation procedure is as follows. Take a realization of the random phases, construct the evolution matrix and obtain the quasienergies $\left\{\omega_{i}\right\}$. Put them in descending order and calculate the level spacings $s_{i}=\left(\omega_{i}-\omega_{i-1}\right) / \delta$, where $\delta$ is the average of $s_{i}$. Repeat this procedure sufficient times so that more than $5 \times 10^{4}$ level spacings are collected for a given $E$ and $J$. The level-spacing distribution function $P(s)$ is thus obtained numerically.

\section{Numerical results and discussions}

\subsection{Analysis of the level-spacing spectrum}

We shall analyse the numerical results of the level-spacing distribution function $P(s)$ in order to provide evidence for the existence of extended state bands in our model. Due to the chirality nature of the drifting motion, time-reversal symmetry is absent from our semiclassical network model. Then, according to the RMT [42], $P(s)$ should be the GUE distribution, 

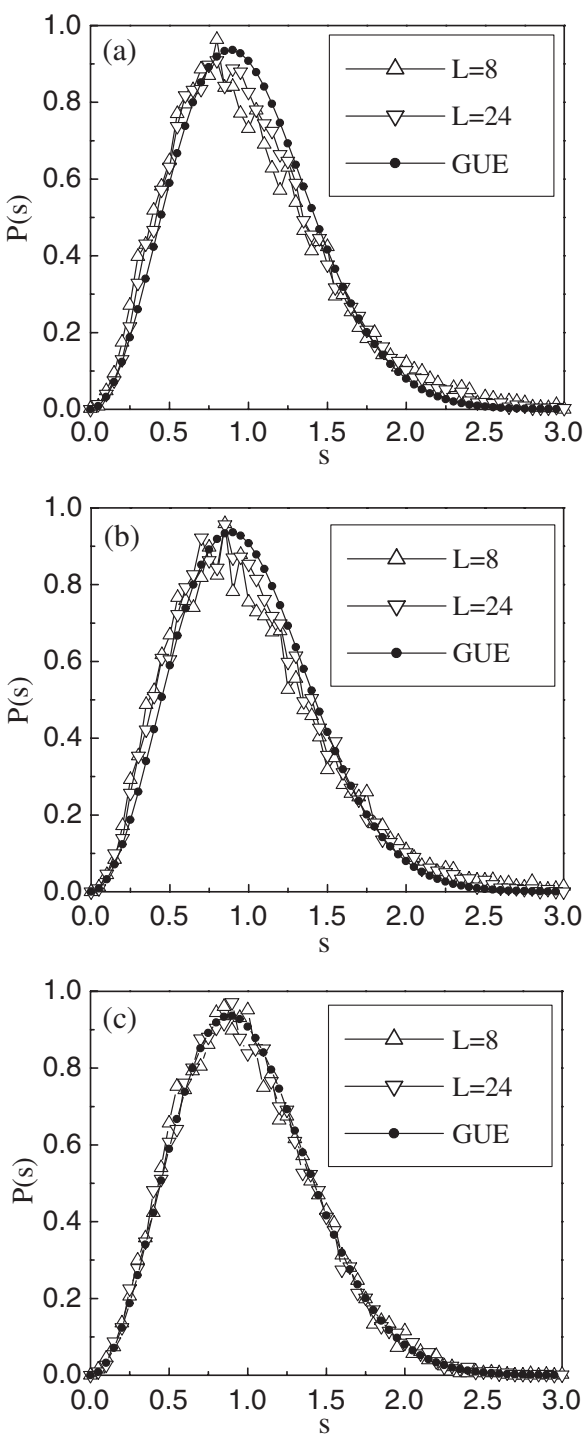

Figure 5. $P(s)$ versus $s$ for $L=8,24$ and $P_{\mathrm{GUE}}(s)$. (a) $E=0$ and $J=0.1$; (b) $E=0.02$ and $J=0.1$; (c) $E=0.5$ and $J=1.5$.

$P_{\mathrm{GUE}}(s)=32 \pi^{-2} s^{2} \exp \left(-4 s^{2} / \pi\right)$, for extended states, and the PE distribution $P_{\mathrm{PE}}(s)$ for localized states. Since the overall shape of the GUE distribution is quite different from that of the PE distribution, one may use $P(s)$ to distinguish an extended state from a localized state. Figure 5 is $P_{\mathrm{GUE}}(s)$ and $P(s)$ for $(E=0, J=0.1)(\mathrm{a}),(E=0.02, J=0.1)$ (b), and ( $E=0.5, J=1.5)$ (c) with $L=8,12,16,20,24$. Figure 6 is $P(s)$ for $(E=0.0, J=0.7)$ (a), $(E=0.02, J=0.7)$ (b) and $(E=0.5, J=0.5)$ (c).

The overall shape of these curves has some common features. All curves have a vanishing value when $s$ goes to zero. At small $s$ they increase with $s$ and reach a peak at some intermediate $s$. Then they decrease monotonically to zero with increasing $s$. These features are the same as those for $P_{\mathrm{GUE}}(s)$ [45]. Thus they all look like $P_{\mathrm{GUE}}(s)$ at first glance. This raises the question 

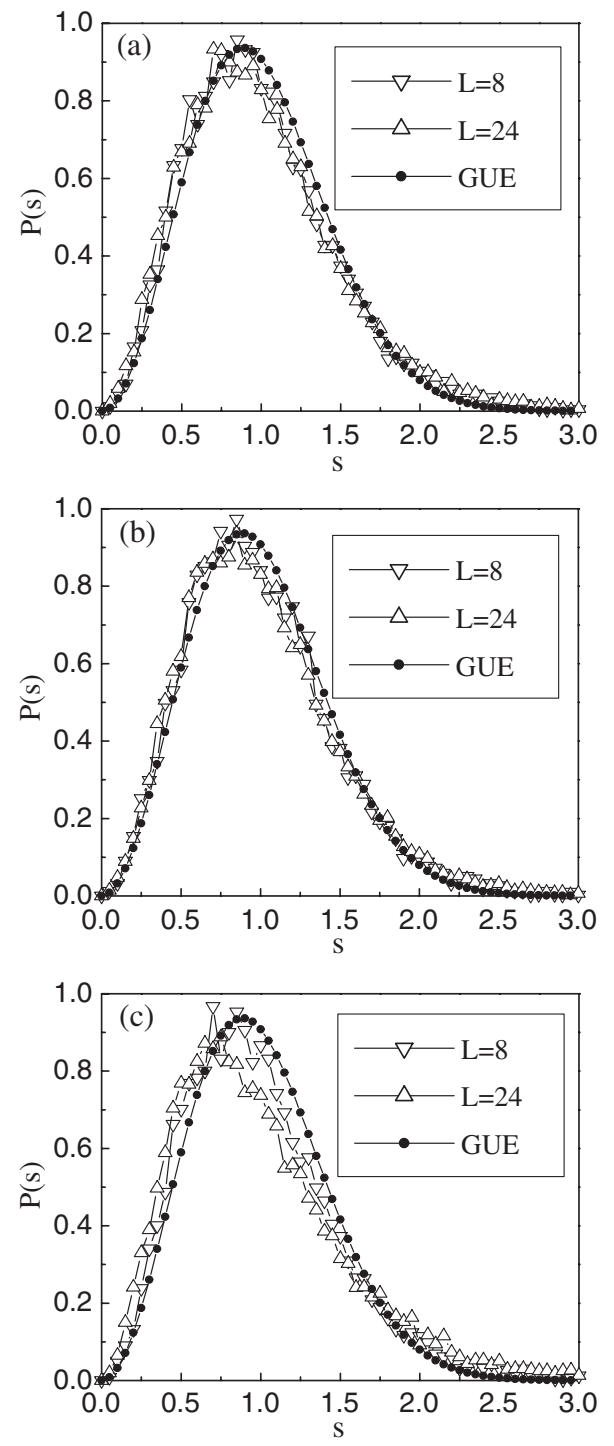

Figure 6. $P(s)$ versus $s$ for $L=8,24$ and $P_{\mathrm{GUE}}(s)$. (a) $E=0$ and $J=0.7$; (b) $E=0.02$ and $J=0.7$; (c) $E=0.5$ and $J=0.5$.

of how to distinguish numerically extended states from localized states. As a simple way, it is natural to expect that $P(s)$ for an extended state approaches $P_{\mathrm{GUE}}(s)$ while that for a localized state should deviate from $P_{\mathrm{GUE}}(s)$ as $L$ increases. Indeed, as $L$ increases, curves in each subfigure of figure 5 approach $P_{\mathrm{GUE}}(s)$ while those in figure 6 show the opposite tendency. Thus we can use this different tendency of $P(s)$ to distinguish extended states from localized states. We shall show quantitatively that such opposite tendency for extended states and localized states also exists in several other characteristic quantities.

Let us first consider a characteristic quantity $I_{0}$ defined by $I_{0}=\int s^{2} P(s) \mathrm{d} s / 2$. It is commonly used to characterize the overall shape of $P(s)$ and to examine the localization property [45]. It is well known that $I_{0}=1$ for localized states while $I_{0}<1$ for extended 

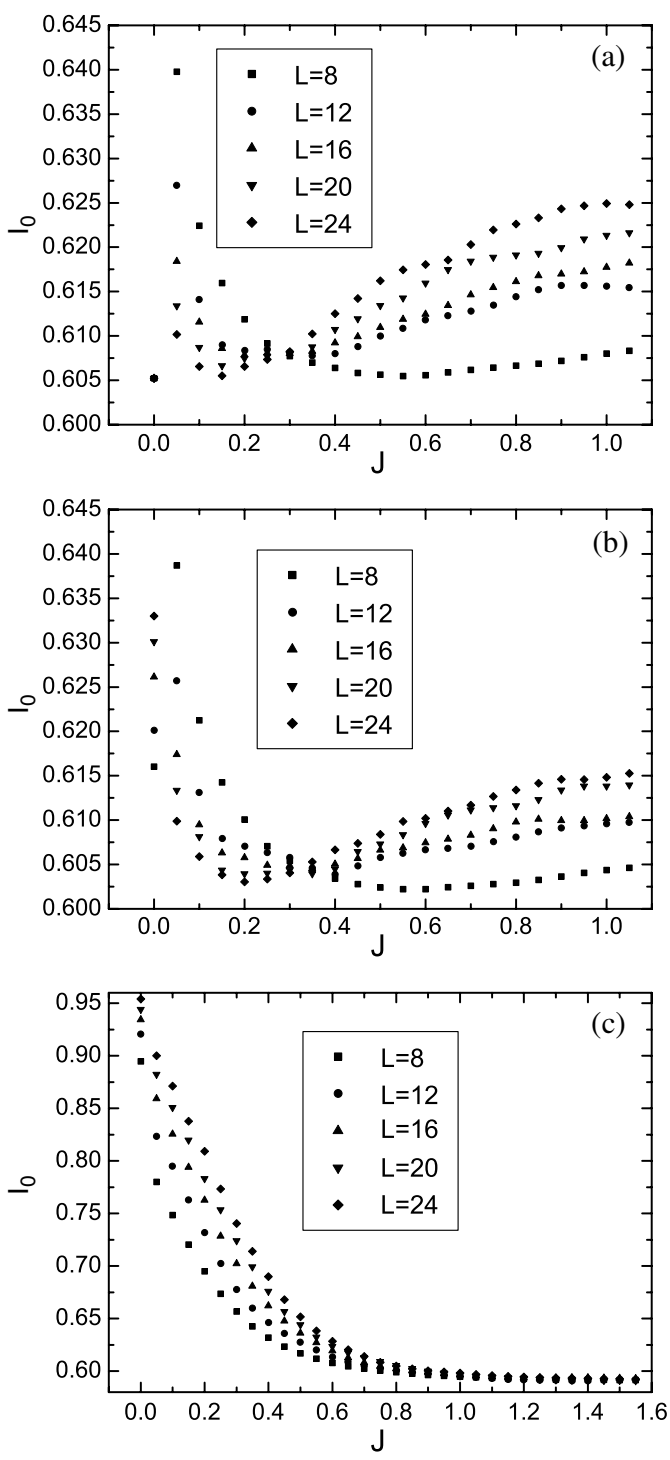

Figure 7. $I_{0}$ versus $J$ for $L=8,12,16,20,24$, (a) $E=0$; (b) $E=0.02$; (c) $E=0.5$.

states [42]. Thus, the following simple criterion is employed: a state is localized if its $I_{0}$ increases and approaches 1 as $L$ increases. Otherwise, it is extended. Curves in figure 7 are $I_{0}$ versus mixing strength $J$ for $E=0$ (a); 0.02 (b); and 0.5 (c) for $L=8,12,16,20,24$. Figure 7(b) shows that the state of $E=0.02$ is localized at zero mixing and extended at small $J$. Then it is localized again after $J$ passes a particular $J_{\mathrm{c}}$ where $I_{0}$ of different $L$ cross. For the state of $E=0$ at the lower band centre shown in figure 7(a), it is extended at zero mixing. Then, it shows the same feature as the state of $E=0.02$ at small and large $J$. Figure 7(c) shows that the state of $E=0.5$ is always localized at small $J$ and extended only for large $J(>1)$ where all curves of different system sizes tend to merge together.

A fundamental difference between $P(s)$ for a localized state and that for an extended states is its behaviour at small $s$. As $s$ goes to zero, $P(s)$ vanishes for extended states due to level 

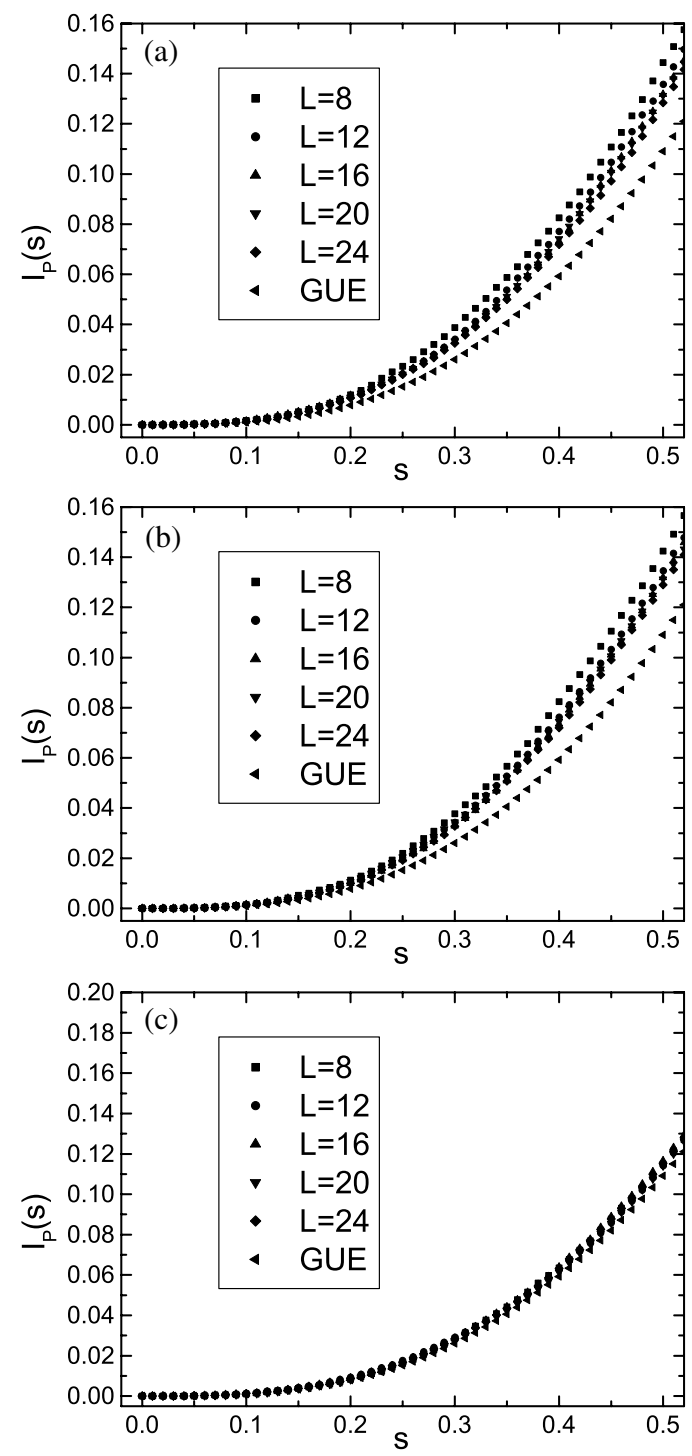

Figure 8. $I_{P}(s)$ versus $s$ for $L=8,12,16,20,24$ and that for $P_{\mathrm{GUE}}(s)$. (a) $E=0$ and $J=0.1$; (b) $E=0.02$ and $J=0.1$; (c) $E=0.5$ and $J=1.5$.

repulsion while it approaches 1 for localized states due to level aggregation [42]. Thus we need to consider the behaviour of $P(s)$ at small $s$ for a further test of the results in the last paragraph. It is convenient to consider a function of integrated level-spacing distribution at small $s$ defined by $I_{P}(s)=\int_{0}^{s} P\left(s^{\prime}\right) \mathrm{d} s^{\prime} . I_{P}(s)$ is the fraction of level spacing smaller than $s$. Although $P(s)$ in most cases of our numerical results is close to the GUE distribution, the level repulsion of extended states and level aggregation of localized states should still be expected at small $s$. This leads to the following criterion: $I_{P}(s)$ at small $s$ should increase (decrease) with $L$ for localized (extended) states. Thus the behaviour of $I_{P}(s)$ at small $s$ can serve as another way of distinguishing extended states from localized ones. Figure 8 shows $I_{P}(s)$ for $(E=0, J=0.1)$ (a), $(E=0.02, J=0.1)$ (b) and $(E=0.5, J=1.5)$ (c) for $L=8,12,16,20,24$ and 

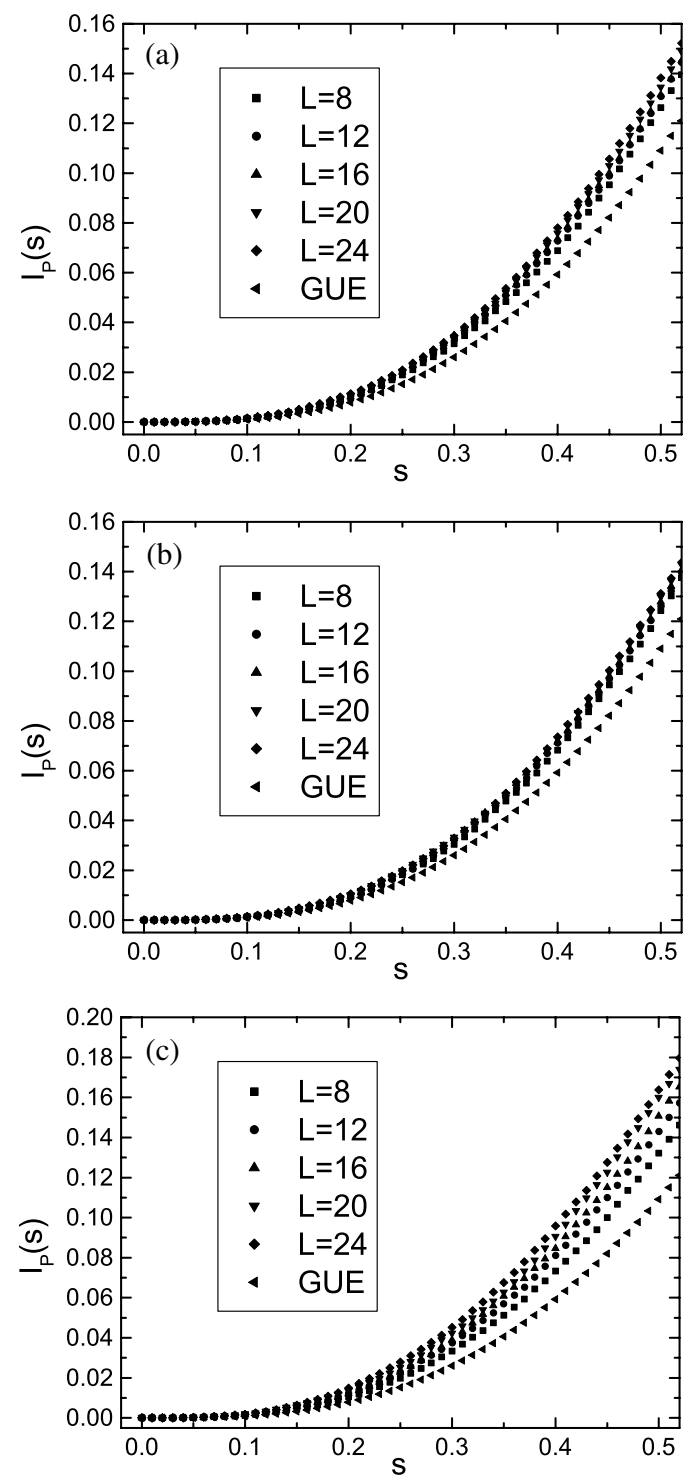

Figure 9. $I_{P}(s)$ versus $s$ for $L=8,12,16,20,24$ and that for $P_{\mathrm{GUE}}(s)$. (a) $E=0$ and $J=0.7$; (b) $E=0.02$ and $J=0.7$; (c) $E=0.5$ and $J=0.5$.

comparison with $I_{P}(s)$ of $P_{\mathrm{GUE}}(s)$. Figure 9 is for $(E=0, J=0.7)$ (a), $(E=0.02, J=0.7)$ (b) and ( $E=0.5, J=0.5)$ (c). One can see clearly that states in figure 8 show the feature of extended states while states in figure 9 are localized. In order to examine an electronic state of fixed energy in the whole range of mixing, we consider $I_{P}(s=0.5)$, the fraction of the level spacings less than 0.5 . We plot the results of $I_{P}(s=0.5)$ versus $J$ at $E=0,0.02$ and 0.5 for $L=8,12,16,20,24$ in figure 10. Similar to the criteria for $I_{P}(s)$, we use the following ones. If $I_{P}(0.5)$ of a state increases with $L$, the state is localized. Otherwise, it is extended. According to this criterion, curves in figure 10 give essentially the same results as those obtained from $I_{0}$ in figure 7 . 

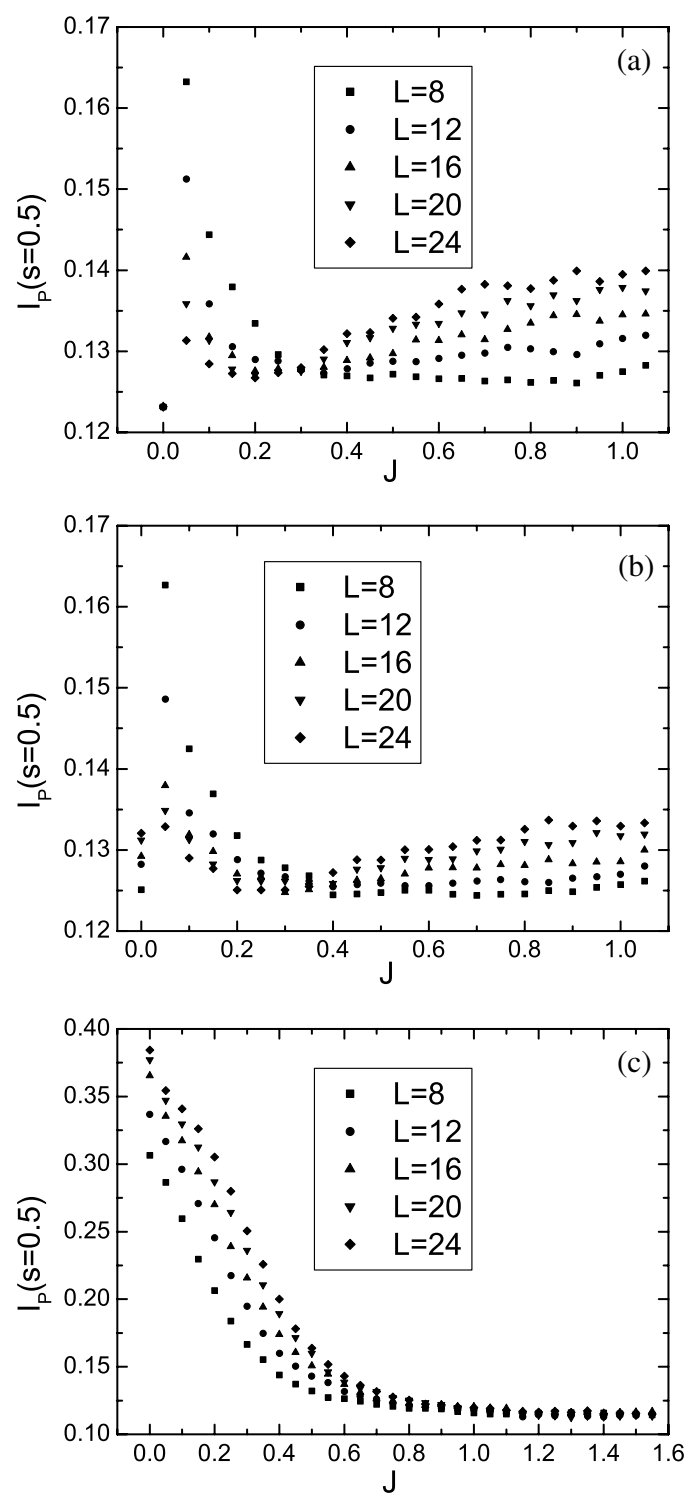

Figure 10. $I_{P}(s=0.5, J)$ versus $J$ for $L=8,12,16,20,24$. (a) $E=0$; (b) $E=0.02$; (c) $E=0.5$.

Let us now turn to the region of large $s$. Since $P_{\mathrm{GUE}}(s)$ decays faster than $P_{\mathrm{PE}}(s)$ at large $s$, the behaviour of $P(s)$ in this region can also be used to differentiate extended and localized states. In this region it is convenient to consider another quantity defined by $F(s)=\int_{s}^{\infty} P(s) \mathrm{d} s=1-I_{P}(s)$. The meaning of $F(s)$ is the integrated fraction of level spacings larger than $s$. Since $F(s)$ of $P_{\mathrm{GUE}}(s)$ is less than that of $P_{\mathrm{PE}}(s)$ at large $s$, we may expect that $F(s)$ at larger $s$ decreases (increases) with $L$ for extended (localized) states. Figure 11 is $F(s)$ for $P_{\mathrm{GUE}}(s)$ and that for $(E=0, J=0.1)(\mathrm{a}),(E=0.02, J=0.1)$ (b), and $(E=0.5, J=1.5)$ (c) with $L=8,12,16,20,24$. Figure 12 is $F(s)$ versus $s$ for $(E=0, J=0.7)(\mathrm{a}),(E=0.02, J=0.7)(\mathrm{b})$, and $(E=0.5, J=0.5)(\mathrm{c})$. In view 

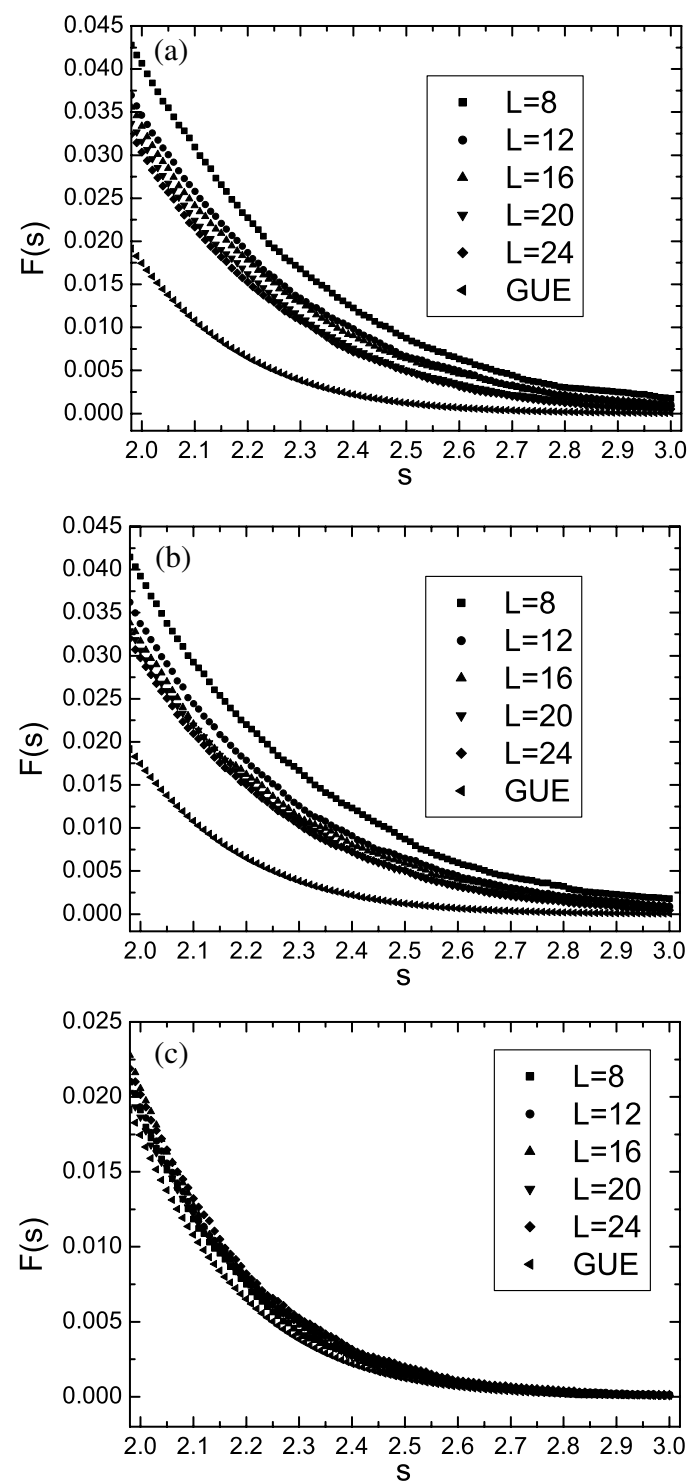

Figure 11. $F(s)$ versus $s$ for $L=8,12,16,20,24$, and that for $P_{\mathrm{GUE}}(s)$. (a) $E=0$ and $J=0.1$; (b) $E=0.02$ and $J=0.1$; (c) $E=0.5$ and $J=1.5$.

of figures 8 and 9, it is clear that the results of $F(s)$ coincide with those of $I_{P}(s)$ concerning the localization property. We also calculate $F(s=2)$ for fixed energy states in the whole range of mixing. As shown above, the same criterion as that for $I_{0}$ and $I_{P}(s=0.5)$ can be employed. The curves of $F(s=2)$ versus $J$ are plotted in figure 13 for $E=0$ (a), $E=0.02$ (b) and $E=0.5$ (c). One can see that they are consistent with the results of $I_{0}$ (figure 7) and $I_{P}(s=0.5)$ (figure 10$)$.

\subsection{Discussion of finite-size effect}

In this subsection, we shall consider the possible influence of the finite-size effect on our numerical results. It is known that localization lengths for $2 \mathrm{D}$ models can exceed $3 \times 10^{4}$ 

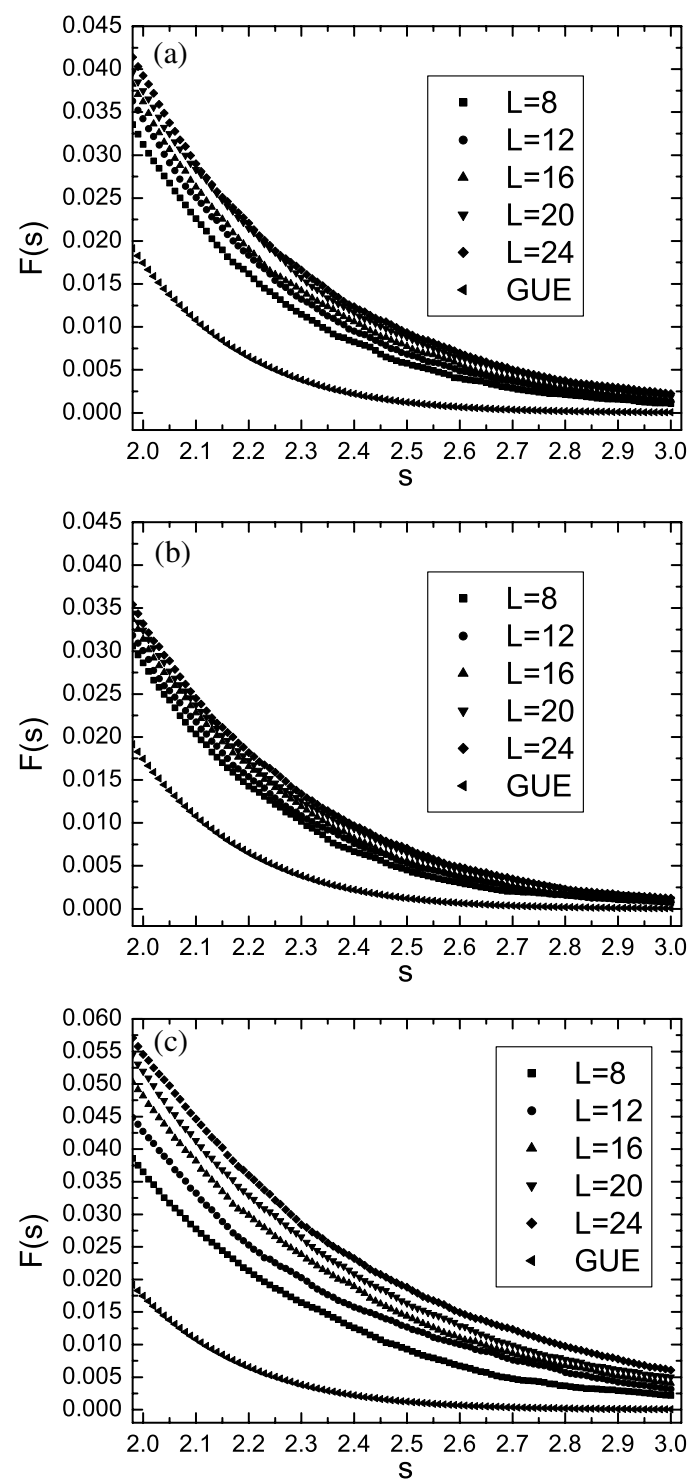

Figure 12. $F(s)$ versus $s$ for $L=8,12,16,20,24$ and that for $P_{\mathrm{GUE}}(s)$. (a) $E=0$ and $J=0.7$; (b) $E=0.02$ and $J=0.7$; (c) $E=0.5$ and $J=0.5$.

lattice spacings, a thousand times larger than the maximum lattice size $L=24$ in our numerical calculation. Therefore, one should worry about the finite-size effect, and question the unsuitability of our simple criteria for the localization property. In the following discussion, we shall only examine the results of the quantity $I_{0}$. Essentially the same discussions can be made for other quantities such as $I_{P}(s)$ and $F(s)$.

Let us first consider the results for $E=0$ and 0.02 in figure 7 . The two cases are quite similar. Curves for different $L$ cross at a single point $J_{\mathrm{c}}$. As $L$ increases, $I_{0}$ decreases and approaches the value for extended states when $J<J_{\mathrm{c}}$, while it increases and approaches the value for localized states when $J>J_{\mathrm{c}}$. A straightforward way of interpreting this behaviour is 

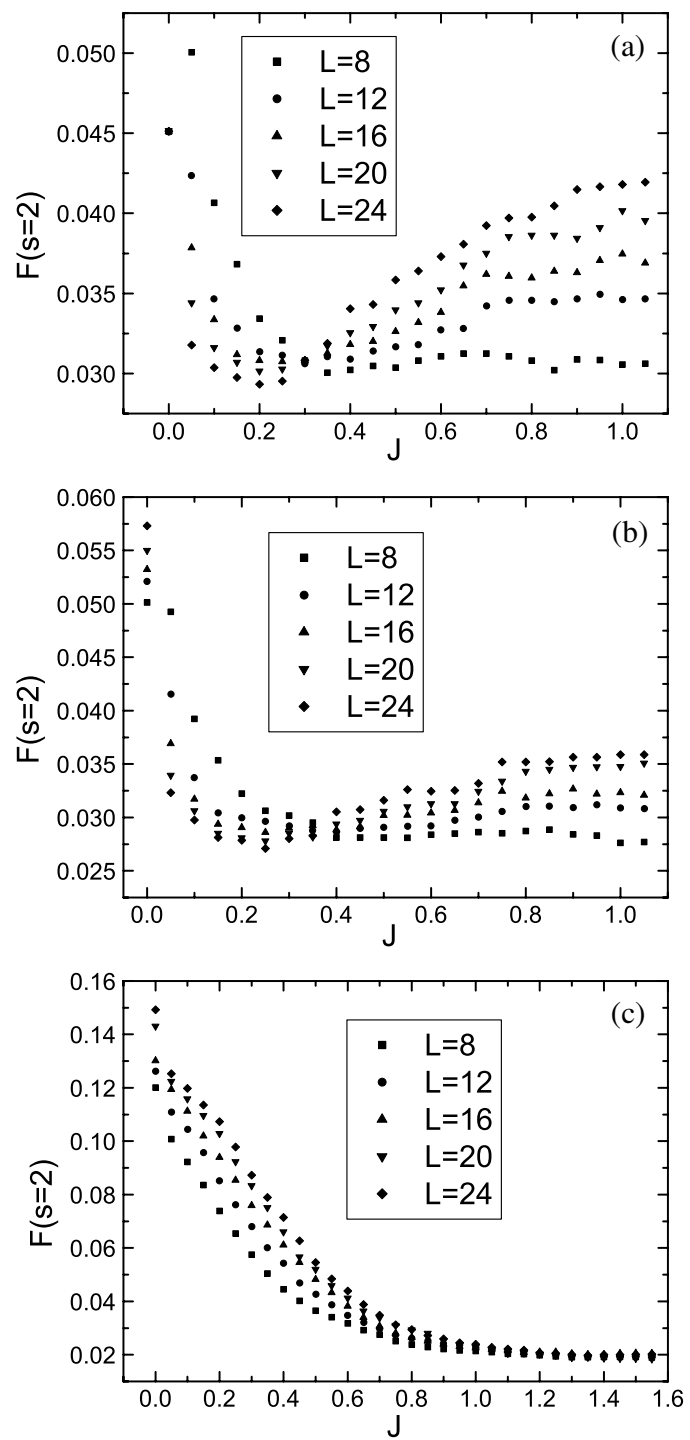

Figure 13. $F(s=2, J)$ versus $J$ for $L=8,12,16,20$, 24. (a) $E=0$; (b) $E=0.02$; (c) $E=0.5$.

that the state exhibits a transition from an extended state in $J<J_{\mathrm{c}}$ to a localized one in $J>J_{\mathrm{c}}$. The correlation length diverges at critical point $J=J_{\mathrm{c}}$ and decreases sharply when $J$ is slightly away from $J_{\mathrm{c}}$. (In a metallic phase the correlation length is small while the localization length is divergent.) Fluctuations at a length scale of the order of the correlation length cause $I_{0}$ to deviate from its thermodynamic-limit value, but $I_{0}$ approaches its thermodynamic-limit values for both $J<J_{\mathrm{c}}$ and $J>J_{\mathrm{c}}$ as lattice size increases. This is a natural explanation of the results. The only finite-size effect in this explanation is rounding behaviour in a region close to the critical point $J=J_{\mathrm{c}}$. This region is normally very narrow because of the sharp drop of the correlation length near $J=J_{\mathrm{c}}$.

Another possible interpretation is to assume that states are always localized in the whole region except at $J=J_{\mathrm{c}}$. In this case, the localization length is equal to the correlation length. 
Then, in order to explain the above behaviour, we have to assume the following features of the localization length: (a) in the region $J<J_{\mathrm{c}}$, the ratio of localization length $\xi(L)$ and system size $L$ should increase with $L$ for small $L$, leading to a false metallic behaviour, while this ratio decreases with $L$ for large $L$, a behaviour for the localized state; (b) in the region $J>J_{\mathrm{c}}, \xi(L) / L$ should always decrease with $L$. In principle, one cannot rule out this possibility without doing calculations for large lattice sizes. However, (a) is too strange to justify. Furthermore, there is no reason to believe why (a) occurs in region $J<J_{\text {c }}$, but not in region $J>J_{\mathrm{c}}$.

For $E=0.5$ in figure 7, localized behaviour is clearly seen for small and intermediate $J$ while the curves of different system size tend to merge at large $J$. Here the finite-size effect should be considered seriously. There are two ways to explain the merging behaviour. One is that a line of critical points exists for large $J$ where the correlation length is always divergent. The other is that the correlation length at the thermodynamic limit is very large yet finite and the merging behaviour is just a finite-size effect. Unlike the case of $E=0$ and 0.02 , both explanations in this case are reasonable. The only way to draw an unambiguous conclusion is to do calculations for large sizes.

However, we can propose a physical picture for the existence of new extended states at $E \sim 0.5$ in the case of strong interband mixing. Assuming that the intraband tunnelling at nodes is negligibly weak for states of $E \sim 0.5$, we have seen already from figure 3(a) that the maximum interband mixing $(\sin \theta=1)$ delocalizes the state which is localized at zero interband mixing. If one views $p=\sin ^{2} \theta$ as the connection probability of two neighbouring loops of opposite chiralities, our two-channel model without intraband tunnellings at nodes is analogous to a bond-percolation problem. It is well known that a percolation cluster exists at $p \geqslant p_{\mathrm{c}}=1 / 2$ or $J \geqslant J_{\mathrm{c}}=1$ for a square lattice [35]. Therefore, an extended state is formed by strong mixing. One hopes that the intraband tunnellings at nodes will only modify the threshold value of the mixing strength. If this picture is correct, the finite-size effect only affects our efforts to determine the accurate value of $J_{\mathrm{c}}$ yet it does not influence the existence of such a critical point.

It should be noted that all the above discussions are based on the single-parameter scaling argument. Suppose that the region of extended states in the thermodynamic limit is vanishing instead of remaining finite; our above numerical results can also be explained by introducing irrelevant length scales and considering their corrections to scaling, as has been pointed out by Pruisken [4] and Huckstein [28]. Thus both our picture of a finite region of extended states and the idea of corrections to scaling are alternative explanations for nonscaling behaviours. Therefore, calculations for larger sizes of system are necessary to draw an unambiguous conclusion for the width of the extended state region in the thermodynamic limit. In a recent theoretical study, Pruisken et al [27] have developed a microscopic theory based on the nonlinear $\sigma$ model to explain the non-scaling behaviours within the assumption of a single critical point. However, in their consideration of the case of long-range correlated disorder which corresponds to the network model, interband mixing, the physical reason for the existence of the finite region of extended states in our picture, is neglected. Therefore, their study does not rule out the possibility of a finite region of extended states.

\subsection{Discussion of localization property}

In the past two subsections, we analysed the global shape of $P(s)$ and its behaviour at small and large $s$ by considering $I_{0}, I_{P}(s)$ and $F(s)$, respectively, and discussed the possible influence of the finite-size effect. Analysis of all these quantities leads to essentially the same conclusion concerning the localization property, as follows. For zero interband mixing, only states at 

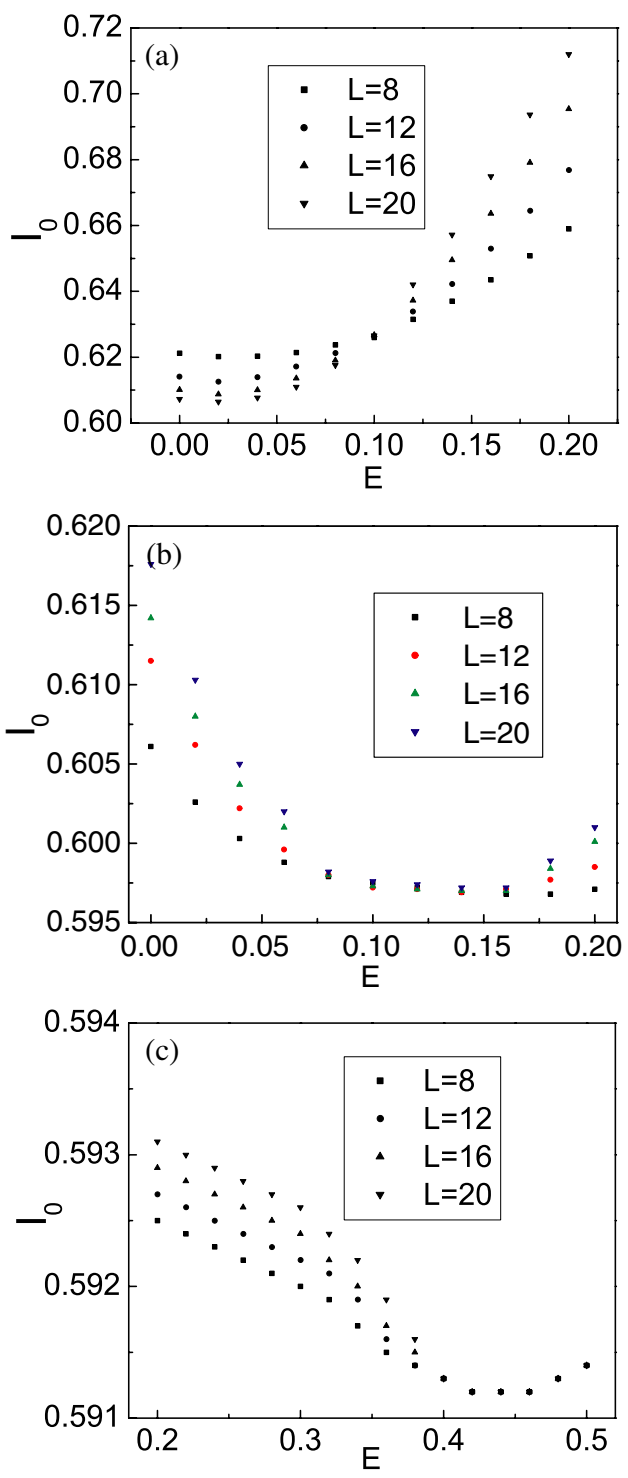

Figure 14. $I_{0}$ versus $E$ for $L=8,12,16,20$. (a) $J=0.1$; (b) $J=0.7$; (c) $J=1.5$.

(This figure is in colour only in the electronic version)

the two LB centres are extended. In the presence of interband mixing, new extended states emerge. States near the LB centres-i.e., $E \sim 0$ - are delocalized by weak interband mixing and localized by strong mixing, with a transition point at some intermediate mixing $J_{\mathrm{c}}$. For states near the region between two LBs-i.e., $E \sim 0.5$ - they are localized at both weak and intermediate mixing and delocalized by strong mixing.

In order to show explicitly the existence of a narrow band of extended states and its evolution with increasing mixing, curves of $I_{0}$ versus $E$ are plotted for three values of $J$ in figure 14. A band of extended states is formed around the LB centre $E \in[0,0.1]$ for $J=0.1$. When $J$ is increased to an intermediate value 0.7 , this band of extended states is lifted up to 


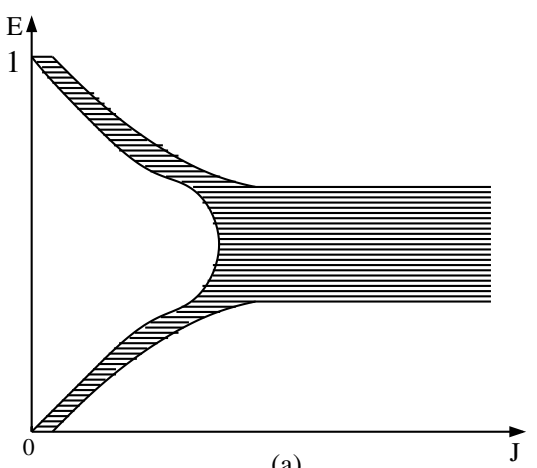

(a)

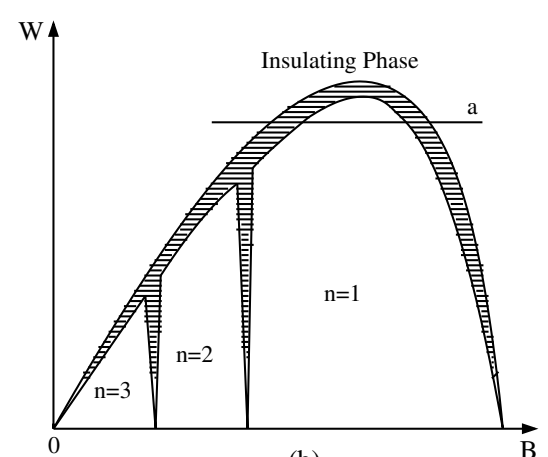

(b)

Figure 15. (a) Topological phase diagram of electron localization in the $E-J$ plane. The shadowed regime is for extended states (metallic phase). (b) Topological QH phase diagram in the $W-B$ plane. $W$ stands for the disorder strength, and $B$ for the magnetic field. The shadowed regime is for the metallic phase. The area indicated by the symbol $n$ is the $n$-plateau IQHE phase. The remaining area is the insulating phase.

$E \in[0.8,1.6]$. For strong mixing, it is further shifted to $E \in[0.4,0.5]$. Thus the band of extended states in the lower LB tends to float up in energy while that of the upper tends to dive down in energy as mixing strength increases. The two bands should finally meet in the middle energy region in the case of strong mixing.

The above results are restricted to the case of two LBs. However, there are an infinite number of LBs in a realistic system. In order to conjecture the situation when an infinite number of LBs is incorporated in our result, we take into account the float-up-merge picture proposed by Sheng et al [12]. We shall expect that a narrow extended band appears in each LB centre for weak interband mixing. Increasing mixing, i.e., increasing disorders or decreasing magnetic field, the extended band in the lowest LB floats up and finally merges with that in the second lowest LB. Then, this extended band will further shift up and merge into that in the third lowest LB, and so on and so forth.

To express our numerical results in the plane of energy and interband mixing, a topological phase diagram shown in figure 15(a) is obtained. In the absence of interband mixing, only the singular energy level at each LB centre is extended. In the presence of interband mixing of opposite chiralities, there are two regimes. At weak mixing, each of the extended states broadens into a narrow band of extended states near the LB centres. With increased mixing, the extended states in the lowest LB shift from the LB centre (see figure 14). These extended states will eventually merge with those from the higher LBs. This shifting of bands of extended states is similar to the shifting of single extended states at LB centres observed in previous studies [5] where emerging of extended bands is missing. At strong mixing, a band of extended states exists between neighbouring LBs where all states are localized without the mixing.

Let us look at the consequences of the above results. For weakly disordered systems in the IQHE regime, the Landau gap is larger than the LB bandwidth. Thus there is no overlap between adjacent LBs. According to the semiclassical picture, electronic states between the two adjacent LBs should be from either the upper or the lower bands with the same chirality in this case. This means that no interband mixing occurs and there is only one extended state in each LB. This may explain why scaling behaviours were observed for plateau transitions in early experiments on clean samples. Interband mixing occurs when the Landau gap is less than the LB bandwidth. Systems of relatively strong disorders in the IQHE regime should correspond to 


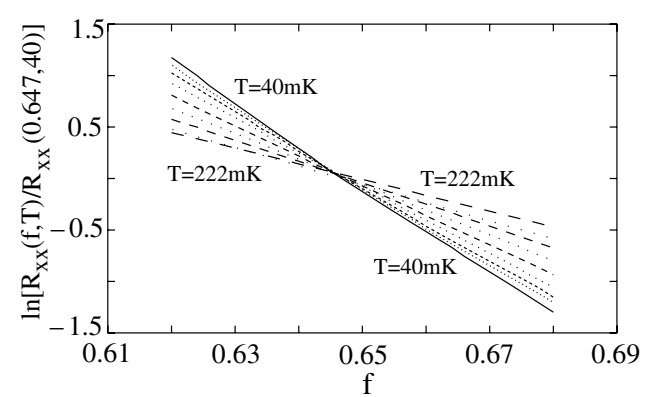

Figure 16. The original experimental data of $\ln \left[R_{x x}(f, T) / R_{x x}(0.647,40 \mathrm{mK})\right]$ in [18] $f$ is the filling factor of LBs and $T$ is the temperature.

this case. As the single extended state at each LB centre broadens into a narrow extended band, a narrow metallic phase emerges between two neighbouring IQHE phases. Thus each plateau transition contains two consecutive quantum phase transitions for strongly disordered systems. The bands of extended states will merge together in strong mixing. This strong mixing regime corresponds to the case when the Landau gap is much smaller than the bandwidth. Since the Landau gap is proportional to the magnetic field, the disordered system should always enter the strong mixing regime before it reaches the weak field insulating phase, regardless of how weak the disorders are. In terms of QH plateau transitions, a direct transition occurs because a narrow metallic phase exists between two QH phases in a weak field. Thus, we propose that a direct transition from an IQHE phase to the insulating phase at weak field is realized by passing through a metallic phase, and it should hold for both weak and strong disordered systems.

Plotting the above results in the plane of disorder and the magnetic field, we obtain a new topological QH phase diagram as shown in figure 15(b). This is similar to the empirical diagram obtained experimentally in [15]. The origin $(W=0, B=0)$ is a singular point. According to the weak localization theory [1], no extended state exists at this point. Differing from existing theories, there exists a narrow metallic phase between two adjacent IQHE phases and between an IQHE phase and an insulating phase.

\subsection{Comparison with previous studies}

In this subsection, we shall compare our results with previous studies. We shall show that the new phase diagram in figure 15 is consistent with the non-scaling experiments [21] where samples are relatively dirty, and interband mixing is strong, corresponding to a process along line $a$ in figure 15(b). The system undergoes two quantum phase transitions each time it moves from the QH insulating phase to IQHE phase of $n=1$ and back to the weak field insulating phase as the magnetic field decreases. To verify this claim, we analysed the original experimental data in [18] according to the assumption of two quantum phase transition points. The experimental data of the logarithm of the longitudinal resistance $\ln \left[R_{x x}(f, T)\right]$ are shown in figure 16 where $f$ is the filling factor of LBs and $T$ is the temperature. According to the theory of continuous transitions, one should obtain

$$
\ln \left[R_{x x}(v, T)\right]=F_{1}\left(S_{1}(f) / T\right)
$$

with $S_{1}(f) \sim\left(f_{\mathrm{c} 1}-f\right)^{z_{1} v_{1}}$ for the region of $f<f_{\mathrm{c} 1}$ while

$$
\ln \left[R_{x x}(v, T)\right]=F_{2}\left(S_{2}(f) / T\right)
$$

with $S_{2}(f) \sim\left(f-f_{\mathrm{c} 2}\right)^{z_{2} v_{2}}$ for the region of $f>f_{\mathrm{c} 2}$. Previous theories predict one single critical point-i.e., $f_{\mathrm{c} 1}=f_{\mathrm{c} 2}$ and $z_{1} v_{1}=z_{2} v_{2}$. But our results suggest two distinct critical 


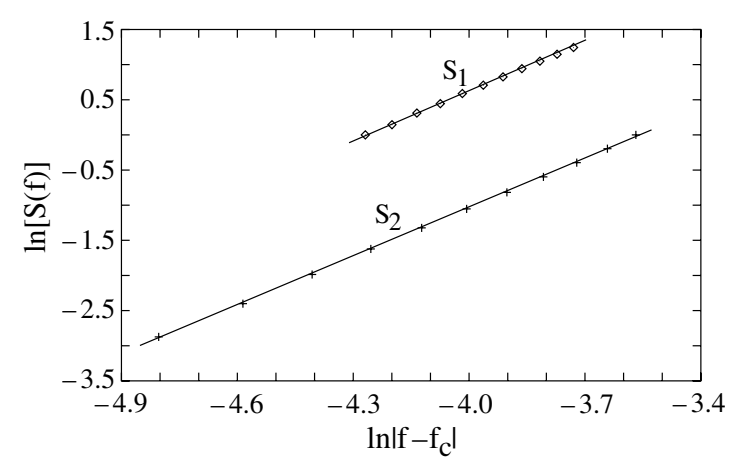

Figure 17. The fitting result of two critical points on the left and the right side. The two straight lines show coincidence with the scaling law. The critical filling factors are $f_{\mathrm{c} 1}=0.6453$ and $f_{\mathrm{c} 2}=0.6477$. The two critical exponents are equal to the value $z v=2.33 \pm 0.01$.

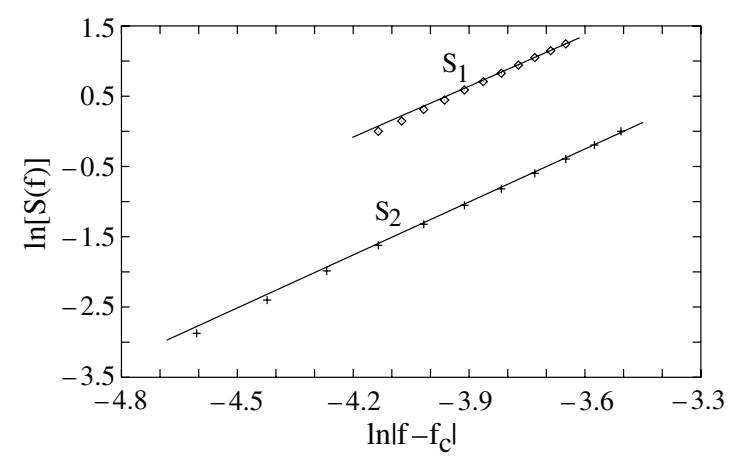

Figure 18. The best fitting result of one single critical point on the left and the right side. The critical filling factor is $f_{\mathrm{c}}=0.646$. The two straight lines illustrate systematic deviations from the scaling law at regions close to the critical point. The average values of the two critical exponents are $z_{1} v_{1}=2.58 \pm 0.02$ and $z_{2} v_{2}=2.60 \pm 0.02$, respectively.

points. By standard scaling analysis, two good scaling behaviours are obtained for two close critical filling factors of $f_{\mathrm{c} 1}=0.6453$ and $f_{\mathrm{c} 2}=0.6477$ as shown in figure 17 . The critical exponents on both the left side and the right side of the transition region are equal to the value $z v=2.33 \pm 0.01$. On the other hand, the fit for one single critical point fails. Figure 18 shows the result of a single critical point at $v_{\mathrm{c}}=0.646$. It is the best fitting result for a single critical point if we require that the two critical exponents are approximately equal and the scaling law is optimally obeyed. The two critical exponents are $z_{1} v_{1}=2.58 \pm 0.02$ and $z_{2} v_{2}=2.60 \pm 0.02$, deviating from the theoretical results $z v \sim 2.33$. One can also see clearly systematic deviations from the scaling law in the region close to the critical point on both sides in figure 18 . This implies that the transition process is governed by two separate critical points instead of one. The regime between the two critical points should correspond to the metallic phase.

It is worth noting that there is another puzzle in the non-scaling experiment which may be solved by our two-critical-point picture. As an example, we consider the experimental data for the transition between the QH insulating phase and the $n=1 \mathrm{IQHE}$ phase. It was shown [21] that the logarithm of the longitudinal resistance $\ln \left[R_{x x}(f, T)\right]$ can be fitted by a linear function of the filling factor $f$ (see figure 16)

$$
\left.\ln \left[R_{x x}(f, T)\right]=\ln \left[R_{x x}\left(f_{\mathrm{c}}, T\right)\right]-\left(f-f_{\mathrm{c}}\right) /(\alpha+\beta T)\right]
$$


where $\alpha$ and $\beta$ are positive constants, $f_{\mathrm{c}}$ is the filling factor where curves of different temperature $T$ cross approximately. Since $\alpha$ is non-zero [21], it leads to the conclusion that $R_{x x}(f, T)$ at the limit of $T=0$ remains finite for every $f$. This is puzzling because it is inconsistent with the theoretical requirement that $R_{x x}(T=0)=\infty$ in the $\mathrm{QH}$ insulating phase, i.e., $f<f_{\mathrm{c}}$, and $R_{x x}(T=0)=0$ in the $n=1$ IQHE phase, i.e., $f>f_{\mathrm{c}}$. This puzzle may be solved as follows. Combine the linear relationship between $\ln \left[R_{x x}(f, T)\right]$ and $f$ for fixed $T$ with our picture of two critical points $f_{\mathrm{c} 1}<f_{\mathrm{c} 2}$; we expect

$$
\ln \left[R_{x x}(f, T)\right]=\ln \left[R_{x x}\left(f_{\mathrm{c} 1}, T\right)\right]-\left(f-f_{\mathrm{c} 1}\right) /\left(A_{1} T^{z \nu}\right)
$$

in the $\mathrm{QH}$ insulating phase, i.e., $f<f_{\mathrm{cl}}$, while

$$
\ln \left[R_{x x}(f, T)\right]=\ln \left[R_{x x}\left(f_{\mathrm{c} 2}, T\right)\right]-\left(f-f_{\mathrm{c} 2}\right) /\left(A_{2} T^{z \nu}\right)
$$

in the $n=1$ IQHE phase, i.e., $f>f_{\mathrm{c} 2}$, where $A_{1}$ and $A_{2}$ are positive constants, and $z$ and $v$ are critical exponents. It is clear that both $R_{x x}(f, T=0)=\infty$ in $f<f_{\mathrm{c} 1}$ and $R_{x x}(f, T=0)=0$ in $f>f_{\mathrm{c} 2}$ are recovered. While a finite value of $R_{x x}(f, T=0)$ in the region $f_{\mathrm{c} 1}<f<f_{\mathrm{c} 2}$ is consistent with our prediction of a metallic phase between the two critical points.

Scaling behaviours of plateau-plateau transitions are observed in recent experiments [29-33]. At a first glance, it seems that these results conflict with both non-scaling experiments and our numerical results. However, there are two important differences between recent scaling experiments and non-scaling experiments. One is that some scaling experiments [29-31] were done deep inside the QH plateau, or far from the plateau transition point, while the non-scaling behaviour was obtained by using data very close to the transition points. In the non-scaling experiments [21], it is known that data not too close to a transition point follow a scaling law. The other is that the samples used in all scaling experiments [29-33] are clean with very high mobility while non-scaling behaviour was observed in relative dirty samples [21-23]. In fact, the mobility in recent scaling experiments is at least one order of magnitude larger than that in early non-scaling experiments. This means that the scaling and non-scaling experiments correspond to regions of vanishing and relatively strong inter-band mixing, respectively. Thus, there is no real conflict between the recent scaling experiments and these early non-scaling experiments. Since our model is valid only when inter-band mixing is considerable, there is also no real conflict between recent scaling experiments and our numerical results.

The two-channel CC model has been used to simulate two degenerate or nearly degenerate spin-resolved Landau subbands with strong interband mixing by Wang et al [7]. They found two distinct critical points, which were related to mixing-induced repulsion [7]. For the nearly degenerate case, they did not consider the states between the two LB centres. For the degenerate case, their study could not discern whether the states between the two critical points are extended or localized. According to our results, a band of extended states is formed around the degenerate LB centre for the degenerate case at strong mixing, while for the nearly degenerate case electronic states between the two LB centres are delocalized by strong mixing. Thus our results are consistent with their results.

One should also notice that two types of metallic phases have been studied extensively in the QH system. One is the composite fermion state at the half-filling in the lowest Landau level (LL) and the other is the stripe state at the half-filled higher LLs. These states are formed by the Coulomb interaction effect in the high mobility samples. They are different from our metallic phase caused by level mixing. Although we have not considered the electron-electron interactions in our study, there is no reason why the delocalization effect of level mixing will be diminished by the Coulomb interaction. Of course, the interaction could lead to a level shift, thus it may modify the band width. 


\section{Conclusions}

In conclusion, we find by numerical calculations within the network model that it is possible that the single extended state at each LB centre in the absence of interband mixing may broaden into a narrow band of extended states when the effect of mixing of states of opposite chirality is taken into account. We also provide a physical picture to show how the mixing of states of opposite chiralities may possibly lead to the existence of extended state bands. Based on the above results, we propose a new phase diagram in which a narrow metallic phase exists between two neighbouring IQHE phases and between an IQHE phase and an insulating phase. This new phase diagram is consistent with non-scaling behaviours observed in recent experiments. A standard scaling analysis on non-scaling experiment data [21] supports our results. However, due to finite-size effects, our numerical results can also be explained based on the assumption of a single critical point. Thus further study on large system size is still needed to conclude whether there are extended state bands in quantum Hall systems in the thermodynamic limit.

\section{Acknowledgments}

This work was substantially supported by a grant from the Research Grant Council of HKSAR, China (project Nos HKUST6153/99P and HKUST6149/00P). GX acknowledges the support of CNSF under grant No 10347101 and a grant from Beijing Normal University. GX and YW also acknowledge the support of CNSF under grant No 90103024. Some calculations were carried out on the SGI of the Physics Department of the Beijing Normal University.

\section{Appendix}

In this appendix, we explicitly construct the evolution matrix $\hat{U}$ for a $2 \times 2$ two-channel CCnetwork model as shown in figure A.1. Periodical boundary conditions in both directions are imposed as explained in section 3. Zs are the wavefunction amplitudes on links. The notations are as follows. $\mathrm{H}$ and $\mathrm{V}$ stand for horizontal and vertical links, respectively. $\mathrm{u}(\mathrm{l})$ is for the upper (lower) LB. $\hat{S}_{i}$ are $S O(4)$ matrices defined in equation (3) describing the tunnelling on nodes, and $\hat{M}_{i}$ are $U(2)$ matrices defined in equations (5) and (6) describing interband mixing. From figure A.1 we can obtain

$$
\begin{aligned}
& \left(\begin{array}{c}
Z_{\mathrm{u}, \mathrm{H}}^{1}(t+1) \\
Z_{1, \mathrm{H}}^{1}(t+1) \\
Z_{1, \mathrm{H}}^{2}(t+1) \\
Z_{\mathrm{u}, \mathrm{H}}^{2}(t+1)
\end{array}\right)=\hat{H}_{1}\left(\begin{array}{c}
Z_{1, \mathrm{~V}}^{1}(t) \\
Z_{\mathrm{u}, \mathrm{V}}^{1}(t) \\
Z_{\mathrm{u}, \mathrm{V}}^{2}(t) \\
Z_{1, \mathrm{~V}}^{2}(t)
\end{array}\right) \\
& \left(\begin{array}{c}
Z_{\mathrm{u}, \mathrm{H}}^{3}(t+1) \\
Z_{1, \mathrm{H}}^{3}(t+1) \\
Z_{1, \mathrm{H}}^{4}(t+1) \\
Z_{\mathrm{u}, \mathrm{H}}^{4}(t+1)
\end{array}\right)=\hat{H}_{2}\left(\begin{array}{c}
Z_{1, \mathrm{~V}}^{3}(t) \\
Z_{\mathrm{u}, \mathrm{V}}^{3}(t) \\
Z_{\mathrm{u}, \mathrm{V}}^{4}(t) \\
Z_{1, \mathrm{~V}}^{4}(t)
\end{array}\right) \\
& \left(\begin{array}{c}
Z_{1, \mathrm{~V}}^{1}(t+1) \\
Z_{\mathrm{u}, \mathrm{V}}^{1}(t+1) \\
Z_{\mathrm{u}, \mathrm{V}}^{2}(t+1) \\
Z_{1, \mathrm{~V}}^{2}(t+1)
\end{array}\right)=\hat{H}_{3}\left(\begin{array}{c}
Z_{\mathrm{u}, \mathrm{H}}^{3}(t) \\
Z_{1, \mathrm{H}}^{3}(t) \\
Z_{1, \mathrm{H}}^{4}(t) \\
Z_{\mathrm{u}, \mathrm{H}}^{4}(t)
\end{array}\right) \\
& \left(\begin{array}{c}
Z_{1, \mathrm{~V}}^{3}(t+1) \\
Z_{\mathrm{u}, \mathrm{V}}^{3}(t+1) \\
Z_{\mathrm{u}, \mathrm{V}}^{4}(t+1) \\
Z_{1, \mathrm{~V}}^{4}(t+1)
\end{array}\right)=\hat{H}_{4}\left(\begin{array}{c}
Z_{\mathrm{u}, \mathrm{H}}^{1}(t) \\
Z_{v, H}^{1}(t) \\
Z_{1, \mathrm{H}}^{2}(t) \\
Z_{\mathrm{u}, \mathrm{H}}^{2}(t)
\end{array}\right),
\end{aligned}
$$




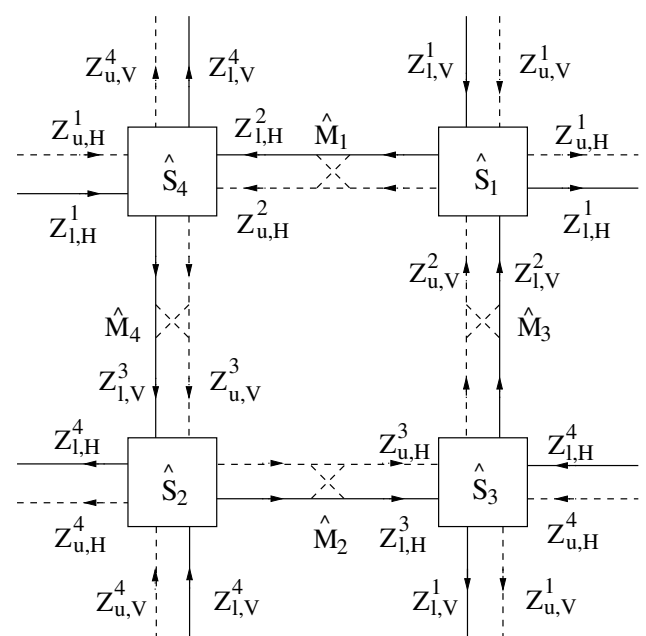

Figure A.1. A two-channel network model of $2 \times 2$ nodes with periodic boundaries along both directions. $Z \mathrm{~s}$ are the wavefunction amplitudes on links. The notations are as follows. $\mathrm{H}$ and $\mathrm{V}$ stand for horizontal and vertical links, respectively. u (1) is for the upper (lower) LB. $\hat{S}_{i}$ are $S O(4)$ matrices describing tunnelling at nodes, and $\hat{M}_{i}$ are $U(2)$ matrices for interband mixing.

with

$$
\begin{aligned}
\hat{H}_{1}=\left(\begin{array}{cc}
\hat{1} & \hat{0} \\
\hat{0} & \hat{M}_{1}
\end{array}\right) \hat{S}_{1} ; & \hat{H}_{2}=\left(\begin{array}{cc}
\hat{M}_{2} & \hat{0} \\
\hat{0} & \hat{1}
\end{array}\right) \hat{S}_{2} ; \\
\hat{H}_{3}=\left(\begin{array}{cc}
\hat{1} & \hat{0} \\
\hat{0} & \hat{M}_{3}
\end{array}\right) \hat{S}_{3} ; & \hat{H}_{4}=\left(\begin{array}{cc}
\hat{M}_{4} & \hat{0} \\
\hat{0} & \hat{1}
\end{array}\right) \hat{S}_{4},
\end{aligned}
$$

where $\hat{1}$ and $\hat{0}$ are the $2 \times 2$ identity and zero matrices, respectively. If we define

$$
\phi_{H}=\left(\begin{array}{c}
Z_{\mathrm{u}, \mathrm{H}}^{1} \\
Z_{1, \mathrm{H}}^{1} \\
Z_{1, \mathrm{H}}^{2} \\
Z_{\mathrm{u}, \mathrm{H}}^{2} \\
Z_{\mathrm{u}, \mathrm{H}}^{3} \\
Z_{1, \mathrm{H}}^{3} \\
Z_{1, \mathrm{H}}^{4} \\
Z_{\mathrm{u}, \mathrm{H}}^{4}
\end{array}\right) ; \quad \phi_{V}=\left(\begin{array}{c}
Z_{1, \mathrm{~V}}^{1} \\
Z_{\mathrm{u}, \mathrm{V}}^{1} \\
Z_{\mathrm{u}, \mathrm{V}}^{2} \\
Z_{1, \mathrm{~V}}^{2} \\
Z_{1, \mathrm{~V}}^{3} \\
Z_{\mathrm{u}, \mathrm{V}}^{3} \\
Z_{\mathrm{u}, \mathrm{V}}^{4} \\
Z_{1, \mathrm{~V}}^{4}
\end{array}\right),
$$

then the evolution equation is

$$
\left(\begin{array}{l}
\phi_{\mathrm{H}}(t+1) \\
\phi_{\mathrm{V}}(t+1)
\end{array}\right)=\hat{U}\left(\begin{array}{l}
\phi_{\mathrm{H}}(t) \\
\phi_{\mathrm{V}}(t)
\end{array}\right) .
$$

The evolution operator $\hat{U}$ is

$$
\hat{U}=\left(\begin{array}{cccc}
\hat{0} & \hat{0} & \hat{0} & \hat{H}_{1} \\
\hat{0} & \hat{0} & \hat{H}_{2} & \hat{0} \\
\hat{H}_{3} & \hat{0} & \hat{0} & \hat{0} \\
\hat{0} & \hat{H}_{4} & \hat{0} & \hat{0}
\end{array}\right)
$$

where $\hat{0}$ is the $4 \times 4$ zero matrix. It has the structure of equation (9). 


\section{References}

[1] Abrahams E, Anderson P W, Licciardello D C and Ramakrishnan T V 1979 Phys. Rev. Lett. 42673

[2] Pruisken A M M 1987 The Quantum Hall Effect ed R E Prange and S M Girvin (New York: Springer)

[3] Wei H P, Tsui D C, Paalanen M P and Pruisken A M M 1988 Phys. Rev. Lett. 611294

[4] Pruisken A M M 1988 Phys. Rev. Lett. 611297

[5] Kivelson S, Lee D H and Zhang S C 1992 Phys. Rev. B 462223

[6] Liu D Z, Xie X C and Niu Q 1996 Phys. Rev. Lett. 76975 Xie X C et al 1996 Phys. Rev. B 544966

[7] Wang Z Q, Lee D H and Wen X G 1994 Phys. Rev. Lett. 722454

[8] Yang K and Bhatt R N 1996 Phys. Rev. Lett. 761316

[9] Shahar D, Tsui D C and Cunningham J E 1995 Phys. Rev. B 52 R14372 Song S-H, Shahar D, Tsui D C, Xie Y H and Monroe D 1997 Phys. Rev. Lett. 782200

[10] Galstyan A G and Raikh M E 1997 Phys. Rev. B 561422

[11] Sheng D N and Weng Z Y 1997 Phys. Rev. Lett. 78318 Sheng D N and Weng Z Y 1998 Phys. Rev. Lett. 80580

[12] Sheng D N, Weng Z Y and Wen X G 2000 Preprint cond-mat/0003117

[13] Haldane F D M and Yang K 1997 Phys. Rev. Lett. 78298

[14] Jiang H W et al 1993 Phys. Rev. Lett. 711439

[15] Wang T K et al 1994 Phys. Rev. Lett. 72709

[16] Glozman I, Johnson C E and Jiang H W 1995 Phys. Rev. Lett. 74594

[17] Kravchenko S V, Mason W E, Fureaux J E and Pudalov V M 1995 Phys. Rev. Lett. 75910

[18] Song S-H, Shahar D, Tsui D C, Xie Y H and Monroe D 1997 Phys. Rev. Lett. 782200

[19] van Schaijk R T F, de Visser A, Olsthoorn S M, Wei H P and Pruisken A M M 2000 Phys. Rev. Lett. 841567

[20] Wang X R, Xie X C, Niu Q and Jain J 2000 Preprint cond-mat/0008411

[21] Hilke M, Shahar D, Song S H, Tsui D C, Xie Y H and Monroe D 1997 Phys. Rev. B 5615545 Shahar D, Hilke M, Li C C, Tsui D C, Sondhi S L, Cunningham J E and Razeghi M 1998 Solid State Commun. 10719

[22] Balaban N Q, Meirav U and Bar-Joseph I 1998 Phys. Rev. Lett. 814967

[23] Shashkin A A, Dolgopolov V T and Kravchenko G V 1994 Phys. Rev. B 4914486

[24] Khmelnitskii D E 1984 Phys. Lett. A 106182

[25] Laughlin R B 1984 Phys. Rev. Lett. 522304

[26] Xiong G, Wang S D, Niu Q, Tian D C and Wang X R 2001 Phys. Rev. Lett. 87216802

[27] Pruisken A M M, Skoric B and Baranov M A 1999 Phys. Rev. B 6016838

[28] Huckstein B 2000 Phys. Rev. Lett. 843141 Huckstein B 1995 Rev. Mod. Phys. 67357

[29] Hohls F, Zeitler U and Haug R J 2001 Phys. Rev. Lett. 865124

[30] Hohls F, Zeitler U and Haug R J 2002 Phys. Rev. Lett. 88036802

[31] Hohls F, Zeitler U, Haug R J, Meisels R, Dybko K and Kuchar F 2002 Phys. Rev. Lett. 89276801

[32] Ponomarenko et al 2004 Physica E 22236

[33] Li W et al 2005 Phys. Rev. Lett. 94206807

[34] Chalker J T and Coddington P D 1988 J. Phys. C: Solid State Phys. 212665

[35] Stauff D and Aharony A 1994 Introduction to Percolation Theory (London: Taylor and Francis)

[36] Lee D K K and Chalker J T 1994 Phys. Rev. Lett. 721510

[37] Kagalovsky V, Horovitz B and Avishiai Y 1997 Phys. Rev. B 557761

[38] Fertig H A and Halperin B I 1987 Phys. Rev. B 367969

[39] Xie X C, Wang X R and Liu D Z 1998 Phys. Rev. Lett. 803563

[40] Zharekeshev I Kh and Kramer B 1997 Phys. Rev. Lett. 79717 Potempa H and Schweitzer L 1999 Ann. Phys., Lpz. 8 Si209 and references therein

[41] Batch M and Schweitzer L 1996 Preprint cond-mat/9608148 Ono Y, Ohtsuki T and Kramer B 1996 J. Phys. Soc. Japan 651734

[42] Mehta M L 1991 Random Matrices 2nd edn (New York: Academic)

[43] Klesse R and Metzler M 1997 Phys. Rev. Lett. 79721

[44] Fertig H A 1988 Phys. Rev. B 38996

[45] Metzler M and Varga I 1998 J. Phys. Soc. Japan 671856 